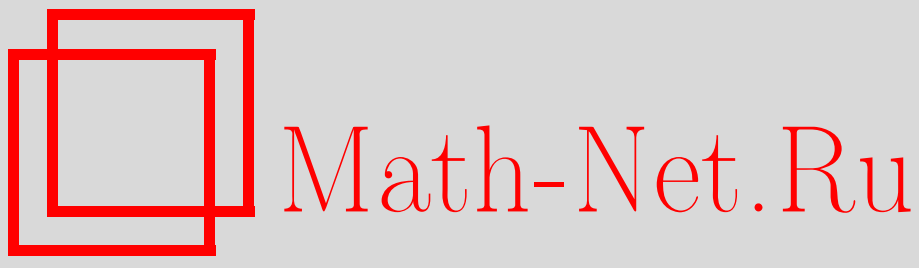

М. И. Вишик, С. В. Зелик, Регулярный аттрактор нелинейной эллиптической системы в цилиндрической области, Матем. сб., 1999, том 190, номер 6, 23-58

DOI: https://doi.org/10.4213/sm411

Использование Общероссийского математического портала Math-Net.Ru подразумевает, что вы прочитали и согласны с пользовательским соглашением http://www . mathnet.ru/rus/agreement

Параметры загрузки:

IP : 3.95 .254 .165

26 апреля 2023 г., 14:21:59 


\section{Регулярный аттрактор нелинейной эллиптической системы в цилиндрической области}

В полуцилиндре $\Omega_{+}=\mathbb{R}_{+} \times \omega, \omega \subset \mathbb{R}^{n}$, рассматривается система эллиптических уравнений второго порядка

$$
a\left(\partial_{t}^{2} u+\Delta_{x} u\right)-\gamma \partial_{t} u-f(u)=g(t),\left.\quad u\right|_{\partial \omega}=0,\left.u\right|_{t=0}=u_{0},(t, x) \in \Omega_{+}, \quad(*)
$$

где $u=\left(u^{1}, \ldots, u^{k}\right)$ - неизвестная векторная функция, $a$ и $\gamma$ - заданные положительно определенные самосопряженные $(k \times k)$-матрицы, $f$ и $g(t)=g(t, x)$ - заданные функции. При выполнении некоторых естественных условий на матрицы $a, \gamma$, нелинейную функцию $f$ и правую часть $g$ доказано, что краевая задача $(*)$ имеет единственное решение, принадлежащее пространству $W_{\text {loc }}^{2, p}\left(\Omega_{+}, \mathbb{R}^{k}\right), p>(n+1) / 2$, и ограниченное при $t \rightarrow \infty$. Кроме того, доказано, что в классе таких решений задача $(*)$ эквивалентна некоторой эволюционной задаче в пространстве "начальных" условий $u_{0} \in V_{0} \equiv \operatorname{Tr}_{t=0} W_{\text {loc }}^{2, p}\left(\Omega_{+}, \mathbb{R}^{k}\right)$. В потенциальном случае $\left(f=\nabla_{x} P, g(t, x) \equiv g(x)\right)$ показано, что полугруппа $S_{t}: V_{0} \rightarrow V_{0}$, порождаемая задачей $(*)$, обладает аттрактором в пространстве $V_{0}$, который в случае общего положения представляется в виде конечного объединения конечномерных неустойчивых многообразий $\mathscr{M}^{+}\left(z_{i}\right)$, соответствующих стационарным точкам $z_{i}$ полугрупшы $S_{t}\left(S_{t} z_{i}=z_{i}\right)$. Кроме того, получена явная формула для вычисления размерностей этих многообразий.

Библиограффия: 20 названий.

\section{Введение}

В полуцилиндре $\Omega_{+}=\mathbb{R}_{+} \times \omega$, где $\omega$ - ограниченная область в $\mathbb{R}^{n}$ с достаточно гладкой границей, рассматривается следующая квазилинейная эллиптическая система второго порядка:

$$
\left\{\begin{array}{l}
a\left(\partial_{t}^{2} u+\Delta_{x} u\right)-\gamma \partial_{t} u-f(u)=g(t) \\
\left.u\right|_{t=0}=u_{0},\left.\quad u\right|_{\partial \omega}=0
\end{array}\right.
$$

Здесь $u=u(t, x)=\left(u^{1}, \ldots, u^{k}\right)$ - неизвестная векторная функция, $g(t)=g(t, x)$ и $f(u)$ - заданные функции, $(t, x) \in \Omega_{+}, \Delta_{x}$ - оператор Лапласа по переменным

Работа выполнена при частичной поддержке Российского фонда фундаментальных исследований (грант № 96-01-00354) и фонда CRDF (грант № RM-1-186). 
$x=\left(x_{1}, \ldots, x_{n}\right), \gamma=\gamma^{*}$ и $a=a^{*}$-положительно определенные самосопряженные матрицшы $\left(\gamma, a \in L\left(\mathbb{R}^{k}, \mathbb{R}^{k}\right)\right)$

$$
0<a_{-} \mathrm{Id} \leqslant a \leqslant a_{+} \mathrm{Id}, \quad 0<\gamma_{-} \mathrm{Id} \leqslant \gamma \leqslant \gamma_{+} \mathrm{Id}
$$

Предполагается, что нелинейная функция $f(u)$ удовлетворяет условиям

1. $f \in C^{1}\left(\mathbb{R}^{k}, \mathbb{R}^{k}\right)$;

2. $f(u) . u \geqslant-C \quad \forall u \in \mathbb{R}^{k}$;

3. $f^{\prime}(u) \geqslant-K, \quad K \geqslant 0, \quad \forall u \in \mathbb{R}^{k}$.

Здесь и далее через $u . v$ обозначается скалярное произведение в $\mathbb{R}^{k}$.

Правая часть $g$ предполагается принадлежашей пространству $L_{p}\left(\Omega_{T}\right)$ для любого $T \geqslant 0\left(\Omega_{T} \equiv[T, T+1] \times \omega\right)$ и некоторого $p>\max \{2,(n+1) / 2\}$ и имеющей конечную норму

$$
|g|_{b}=\sup _{T \in \mathbb{R}_{+}}\left\|g, \Omega_{T}\right\|_{0, p}<\infty .
$$

Здесь и далее $\left\|v, \Omega_{T}\right\|_{l, p} \equiv\|v\|_{W_{l, p}\left(\Omega_{T}\right)}$, а символ $W_{l, p}$ обозначает соболевскоепространство обобшенных функций, производные которых до порядка $l$ включительно принадлежат пространству $L_{p}$ (см. [1]).

Под решением задачи (0.1) понимается обобшенное решение $u \in W_{2, p}\left(\Omega_{T}\right)$ для любого $T \geqslant 0$, обладаюшее конечной нормой

$$
\|u\|_{b}=\sup _{T \in \mathbb{R}_{+}}\left\|u, \Omega_{T}\right\|_{2, p}<\infty
$$

Предполагается также, что "начальное" условие $u_{0}$ принадлежит пространству $V_{0}$ следов функций из $W_{2, p}\left(\Omega_{+}\right)$при $t=0$ (см. [2]):

$$
V_{0}=\operatorname{Tr}_{t=0}\left(W_{2, p}\left(\Omega_{+}\right) \cap\left\{\left.u\right|_{\partial \omega}=0\right\}\right)=W_{2-1 / p, p}(\omega) \cap W_{1, p}^{0}(\omega) .
$$

Системы уравнений вида (0.1) возникают, например, при исследовании положений равновесия или решений типа бегуших волн для соответствуюших эволюционных уравнений (см. замечание 5.3).

Краевые задачи вида (0.1) при различных предположениях относительно нелинейной функции $f$ и правой части $g$ исследовались в работах [3]-[11].

В настоящей работе основное внимание уделяется случаю, когда задача (0.1) имеет единственное решение. Для этого предполагается, что сушествует число $\lambda_{0}>0$ такое, что операторный пучок

$$
L_{K}(\lambda)=a \lambda^{2}-\gamma \lambda+a \Delta_{x}+K: W_{1,2}^{0}(\omega) \rightarrow W_{-1,2}(\omega)
$$

удовлетворяет условию

$$
-\left(L_{K}\left(\lambda_{0}\right) v, v\right)>0 \text { для любого } v \in W_{1,2}^{0}(\omega), v \neq 0 .
$$


Как показано в лемме 1.1, условие (0.8) заведомо вьполнено, если

$$
\gamma^{2}>4 a_{+}\left(K-a_{-} \mu_{1}\right) \mathrm{Id} .
$$

Здесь через $\mu_{1}>0$ обозначено первое собственное число оператора Лапласа в области $\omega$ с граничными условиями Дирихле.

Траекторный аттрактор системы (0.1) без предположения единственности построен в [4].

Центральную роль при исследовании поведения решений задачи (0.1) играет следуюшая теорема, доказанная в $\S 2$ и $§ 3$.

Теорема 1. 1. Пусть выполнены перечисленные выше условия. Тогда задача (0.1) имеет единственное решение, удовлетворяющее (0.5), и справедлива оценка

$$
\left\|u, \Omega_{T}\right\|_{2, p} \leqslant Q\left(\left\|u_{0}\right\|_{V_{0}}\right) e^{-\alpha T}+Q\left(|g|_{b}\right) .
$$

Здесь константа $\alpha>0$ и монотонная функиия $Q: \mathbb{R}_{+} \rightarrow \mathbb{R}_{+}$не зависят от начального условия $u_{0}$.

2. Пусть $S_{t}: V_{0} \rightarrow V_{0}-$ разрешающий оператор задачи $(0.1)\left(S_{t} u(0)=u(t)\right)$. Тогда $S_{t} \in C^{1}\left(V_{0}, V_{0}\right)$ при любом $t \geqslant 0$ и его производная Фреше $D_{u_{0}} S_{t}\left(u_{0}\right)$ удовлетворяет оценке

$$
\left\|D_{u_{0}} S_{t}\left(u_{0}\right)\right\|_{L\left(V_{0}, V_{0}\right)} \leqslant Q\left(\left\|u_{0}\right\|_{V_{0}}\right) e^{\left(\lambda_{0}-\alpha\right) t}
$$

для некоторого полоэсительного $\alpha$.

Кроме того, в $§ 3$ доказано, что задача (0.1) эквивалентна следующей эволюционной задаче в пространстве $V_{0}$ :

$$
\left\{\begin{array}{l}
\partial_{t} u+\left(-\Delta_{x}\right)^{1 / 2} u=F(t, u)+G(t), \\
\left.u\right|_{t=0}=u_{0}, \quad u \in C_{b}\left(\mathbb{R}_{+}, V_{0}\right) \cap C_{b}^{1}\left(\mathbb{R}_{+}, V_{0}^{\prime}\right) .
\end{array}\right.
$$

Здесь $V_{0}^{\prime}=\left(-\Delta_{x}\right)^{1 / 2} V_{0}$, функция $G \in C\left(\mathbb{R}_{+}, V_{0}^{\prime}\right)$, а нелинейный оператор $F \in$ $C\left(\mathbb{R}_{+} \times V_{0}, V_{0}\right)$ (см. теорему 3.3$)$.

Параграфы 4 и 5 настоящей работы посвящены более детальному исследованию “автономного" случая

$$
g(t) \equiv g \in L_{p}(\omega)
$$

В этом случае, как нетрудно проверить, операторы $\left\{S_{t}, t \geqslant 0\right\}$ образуют полугруппу в пространстве $V_{0}$. Стационарные точки этой полугруппы исследованы в 4 . Здесь также приведены необходимые и достаточные условия гиперболичности стационарной точки $z_{0}$, доказана конечность индекса неустойчивости ind $_{z_{0}}$ стационарной точки $z_{0}$ и получены формулы для его вычисления. Кроме того, доказано, что неустойчивое множество $\mathscr{M}^{+}\left(z_{0}\right)$ гиперболической стационарной точки $z_{0}$ (см. определение 4.3 ) обладает структурой $C^{1}$-многообразия, диффеоморфного $\mathbb{R}^{d}, d=\operatorname{ind}_{z_{0}}$.

В $\S 5$ построен аттрактор $\mathscr{A}$ полугруппы $\left\{S_{t}, t \geqslant 0\right\}$, компактньй в пространстве $W_{2, p}(\omega)$. Кроме того, в потенциальном случае

$$
f=-\nabla P, \quad P \in C^{2}\left(\mathbb{R}^{k}, \mathbb{R}^{k}\right)
$$

доказана следующая теорема. 
ТЕОРЕМа 2. Пусть выполнены перечисленные выше условия, и пусть все стационарные точки $\mathscr{R}=\left\{z_{1}, \ldots, z_{N}\right\}$ полугруппь $S_{t}$ являются гиперболическими. Тогда аттрактор $\mathscr{A}$ является регулярным (см. теорему 5.4 и следствия из нее), m.e.

$$
\mathscr{A}=\bigcup_{i=1}^{N} \mathscr{M}^{+}\left(z_{i}\right)
$$

и әкспоненциально притягивающим (см. теорему 5.5 и следствие из нее).

Кроме того,

$$
\operatorname{dim} \mathscr{M}^{+}\left(z_{i}\right)=\operatorname{ind}_{z_{i}}=\#\left\{\lambda \in \sigma\left(a \Delta_{x}-f^{\prime}\left(z_{i}\right)\right), \lambda>0\right\} .
$$

Для эволюционных уравнений, обладающих глобальной функцией Ляпунова, представление аттрактора в виде (0.14) получено в [12], [13].

Параграфф 1 содержит доказательства некоторых вспомогательных результатов относительно линейного уравнения вида (0.1), использующихся в дальнейшем при исследовании уравнения в вариациях для нелинейной задачи (0.1).

\section{§1. Линейное эллиптическое уравнение в полуцилиндре}

Этот параграф̆ посвящен исследованию следующей линейной системы в полуцилиндре $\Omega_{+}=\mathbb{R}_{+} \times \omega\left(\omega \Subset \mathbb{R}^{n}\right)$ :

$$
\left\{\begin{array}{l}
a\left(\Delta_{x} u+\partial_{t}^{2} u\right)-\gamma \partial_{t} u-q(t, x) u=g(t) \\
\left.u\right|_{\partial \omega}=0,\left.\quad u\right|_{t=0}=u_{0}
\end{array}\right.
$$

Здесь $u, g$-векторные функции, $q \in L_{\infty}\left(\Omega_{+}, \mathscr{L}\left(\mathbb{R}^{k}, \mathbb{R}^{k}\right)\right), a$ и $\gamma$-самосопряженные матрицы, причем $a>0$ - положительно определенная матрица. Выберем константу $K \in \mathbb{R}$ так, чтобы

$$
q(t, x) \geqslant-K \text { Id для почти всех }(t, x) \in \Omega_{+} .
$$

Кроме того, предполагается, что пучок (0.7) удовлетворяет условию (0.8) для некоторого $\lambda_{0} \geqslant 0$ и $K$, определенного формулой (1.2), а правая часть $g$ имеет конечную норму

$$
|g|_{\lambda_{0}} \equiv \sup _{T \in \mathbb{R}_{+}} e^{-\lambda_{0} T}\left\|g, \Omega_{T}\right\|_{0, p}<\infty .
$$

Под решением задачи (1.1) понимается его обобщенное решение $u$, принадлежащее пространству $W_{2, p}\left(\Omega_{T}\right)$ для любого $T \geqslant 0$ и имеющее конечную норму

$$
\|u\|_{\lambda_{0}}=\sup _{T \in \mathbb{R}_{+}} e^{-\lambda_{0} T}\left\|u, \Omega_{T}\right\|_{2, p}<\infty .
$$

Начальное условие $u_{0}$ выбирается из пространства $V_{0}$ (см. (0.6)).

ЛЕмма 1.1. Условие (0.8) заведомо выполнено, если $\gamma \geqslant 0 и$

$$
\gamma^{2}>4\left(K-\mu_{1} a_{-}\right) a_{+} \mathrm{Id} .
$$

Здесь через $\mu_{1}>0$ обозначено первое собственное число оператора $-\Delta$ в области $\omega$ с граничными условиями Дирихле. 
ДокАЗАТЕЛЬСтво. Из условия (1.5) следует, что существует число $\gamma_{0} \geqslant 0$ такое, что

$$
\gamma \geqslant \gamma_{0} \text { Id } \quad \text { и } \gamma_{0}^{2}>4\left(K-\mu_{1} a_{-}\right) a_{+} .
$$

Таким образом, $a_{+} \lambda_{0}^{2}-\gamma_{0} \lambda_{0}-\mu_{1} a_{-}+K<0$ при $\lambda_{0}=\gamma_{0} / 2 a_{+}$и, следовательно, для любого $v \in W_{1,2}^{0}(\omega), v \neq 0$,

$$
\begin{aligned}
\left(L_{K}\left(\lambda_{0}\right) v, v\right) & =(a v, v) \lambda_{0}^{2}-(\gamma v, v) \lambda_{0}-(a \nabla v, \nabla v)+(K v, v) \\
& \leqslant\left(a_{+} \lambda_{0}^{2}-\gamma_{0} \lambda_{0}-\mu_{1} a_{-}+K\right)\|v\|_{0,2}^{2}<0 .
\end{aligned}
$$

Здесь и далее $a \nabla v \cdot \nabla v \equiv \sum_{i} a \partial_{x_{i}} v . \partial_{x_{i}} v$. Лемма 1.1 доказана.

Лемма 1.2. Условие (0.8) әквивалентно следующему:

$$
-\left(L_{K}\left(\lambda_{0}\right) v, v\right) \geqslant \varepsilon\|v\|_{1,2}^{2} \text { для некоторого } \varepsilon>0 .
$$

ДокАЗАТЕльСтво. Предположим противное, тогда сушествуют $v_{i} \in W_{1,2}^{0}(\omega)$, $\left\|v_{i}\right\|_{1,2}=1, i \in \mathbb{N}$, такие, что

$$
\left(L_{K}\left(\lambda_{0}\right) v_{i}, v_{i}\right)=-\varepsilon_{i}, \quad \varepsilon_{i} \geqslant 0, \quad \varepsilon_{i} \rightarrow 0 \text { при } i \rightarrow \infty .
$$

Без ограничения общности можно считать, что $v_{i} \neg v_{0} \in W_{1,2}^{0}(\omega)$ в пространстве $W_{1,2}^{0}$ и $v_{i} \rightarrow v_{0}$ в пространстве $H$. Переходя к пределу при $i \rightarrow \infty$ в формуле (1.7), учитывая, что $\left(a \nabla v_{0}, \nabla v_{0}\right) \leqslant \liminf _{i \rightarrow \infty}\left(a \nabla v_{i}, \nabla v_{i}\right)$, получим $\left(L_{K}\left(\lambda_{0}\right) v_{0}, v_{0}\right) \geqslant 0$. Таким образом, согласно условию $(0.8) v_{0}=0$. Переписав соотношение (1.7) в виде

$$
\left(a \nabla v_{i}, \nabla v_{i}\right)=\left(a v_{i}, v_{i}\right) \lambda_{0}^{2}-\left(\gamma v_{i}, v_{i}\right) \lambda_{0}+\left(K v_{i}, v_{i}\right)+\varepsilon_{i}
$$

получим $\left\|v_{i}\right\|_{1,2} \rightarrow 0$ при $i \rightarrow \infty$, что противоречит выбору последовательности $v_{i}$. Лемма 1.2 доказана.

Основным результатом этого параграфа является следующая теорема.

ТеОРема 1.1. Пусть выполнены перечисленные выше условия. Тогда задача (1.1) имеет единственное решение и(t) и справедлива оченка

$$
\left\|u, \Omega_{T}\right\|_{2, p}^{p} \leqslant C e^{p \lambda_{0} T}\left(\left\|u_{0}\right\|_{V_{0}}^{p} e^{-\alpha T}+\int_{\mathbb{R}_{+}} e^{-\alpha|T-s|-p \lambda_{0} s}\|g(s)\|_{0, p}^{p} d s\right)
$$

для некоторого положительного $\alpha$.

Для доказательства этой теоремы нам понадобится ряд вспомогательных утверждений.

ЛЕмма 1.3. Пусть и-решение задачи (1.1). Тогда справедлива оценка

$$
\left\|u, \Omega_{T}\right\|_{1,2}^{2} \leqslant C e^{2 \lambda_{0} T}\left(\left\|u_{0}\right\|_{V_{0}}^{2} e^{-\alpha T}+\int_{\mathbb{R}_{+}} e^{-\alpha|T-s|-2 \lambda_{0} s}\|g(s)\|_{0,2}^{2} d s\right) .
$$


ДокаЗАТЕЛЬСтво. Рассмотрим функцию $v \in W_{2, p}\left(\Omega_{+}\right)$такую, что

$$
\left.v\right|_{t=0}=u_{0},\left.\quad v\right|_{\partial \omega}=0, \quad \operatorname{supp} v \subset \Omega_{0}, \quad\left\|v, \Omega_{+}\right\|_{2, p} \leqslant C\left\|u_{0}\right\|_{V_{0}} .
$$

Введем новую неизвестную функцию $\theta(t)=u(t) e^{-\lambda_{0} t}-v(t)$. Тогда уравнение (1.1) примет вид

$$
\left\{\begin{array}{l}
a \partial_{t}^{2} \theta-\left(\gamma-2 a \lambda_{0}\right) \partial_{t} \theta+\left[a \lambda_{0}^{2}-\gamma \lambda_{0}+a \Delta_{x}-q(t, x)\right] \theta=g_{1}(t), \\
\left.\theta\right|_{\partial \omega}=0,\left.\quad \theta\right|_{t=0}=0
\end{array}\right.
$$

Здесь

$$
g_{1}(t)=g(t) e^{-\lambda_{0} t}-a \partial_{t}^{2} v+\left(\gamma-2 a \lambda_{0}\right) \partial_{t} v-\left[a \lambda_{0}^{2}-\gamma \lambda_{0}+a \Delta_{x}-q(t, x)\right] v .
$$

Умножим уравнение (1.11) скалярно в $\mathbb{R}^{k}$ на функцию $\theta(t) \varphi_{T}(t)$, где $\varphi_{T}(t)=$ $e^{-\alpha|T-t|}, \alpha$ - некоторое достаточно малое положительное число, которое будет определено позднее, и проинтегрируем по области $\Omega_{+}$. Получим:

$$
\begin{aligned}
& \left\langle a \partial_{t}^{2} \theta, \theta(t) \varphi_{T}(t)\right\rangle-\left\langle\left(\gamma-2 a \lambda_{0}\right) \partial_{t} \theta, \theta(t) \varphi_{T}(t)\right\rangle \\
& \quad+\left\langle\left[a \lambda_{0}^{2}-\gamma \lambda_{0}+a \Delta_{x}-q(t, x)\right] \theta, \theta(t) \varphi_{T}(t)\right\rangle=\left\langle g_{1}(t), \theta(t) \varphi_{T}(t)\right\rangle .
\end{aligned}
$$

Здесь символом $\langle\cdot, \cdot\rangle$ обозначено скалярное произведение в $L_{2}\left(\Omega_{+}\right)$. Так как согласно определению решения $u(t)$ функция $\theta(t)$ ограничена при $t \rightarrow+\infty$, т.е.

$$
\|\theta\|_{b}=\sup _{T \geqslant 0}\left\|u, \Omega_{T}\right\|_{2, p}<\infty,
$$

то все выписанные выше интегралы имеют смысл.

Оценим отдельно каждое слагаемое в левой части формулы (1.12). Интегрируя по частям, используя положительную определенность матрицы $a$, оценку

$$
\left|\partial_{t} \varphi_{T}(t)\right| \leqslant \alpha \varphi_{T}(t)
$$

и неравенство Гёльдера, получим:

$$
\begin{aligned}
-\left\langle a \partial_{t}^{2} \theta, \theta(t) \varphi_{T}(t)\right\rangle & =\left\langle a \partial_{t} \theta . \partial_{t} \theta, \varphi_{T}(t)\right\rangle+\left\langle a \partial_{t} \theta . \theta, \partial_{t} \varphi_{T}(t)\right\rangle \\
& \geqslant C\left\langle\left|\partial_{t} \theta\right|^{2}, \varphi_{T}(t)\right\rangle-C_{1} \alpha^{2}\left\langle|\theta|^{2}, \varphi_{T}(t)\right\rangle .
\end{aligned}
$$

Аналогично, используя самосопряженность матриц $a$ и $\gamma$, получим:

$$
\begin{aligned}
\left|\left\langle\left(\gamma-2 a \lambda_{0}\right) \partial_{t} \theta, \theta(t) \varphi_{T}(t)\right\rangle\right| & =\frac{1}{2}\left|\left\langle\partial_{t}\left[\left(\gamma-2 a \lambda_{0}\right) \theta . \theta\right], \varphi_{T}(t)\right\rangle\right| \\
& =\frac{1}{2}\left|-\left\langle\left[\left(\gamma-2 a \lambda_{0}\right) \theta . \theta\right], \partial_{t} \varphi_{T}(t)\right\rangle\right| \\
& \leqslant C_{2} \alpha\left\langle|\theta|^{2}, \varphi_{T}(t)\right\rangle .
\end{aligned}
$$

Из оценки (1.6) следует, что

$$
\begin{aligned}
-\left\langle\left[ a \lambda_{0}^{2}-\gamma \lambda_{0}\right.\right. & \left.\left.+a \Delta_{x}-q(t, x)\right] \theta, \theta(t) \varphi_{T}(t)\right\rangle \\
& \geqslant \varepsilon\left\langle|\nabla \theta|^{2}, \varphi_{T}(t)\right\rangle \geqslant \frac{\varepsilon}{2}\left\langle|\nabla \theta|^{2}, \varphi_{T}(t)\right\rangle+\frac{\varepsilon}{2} \mu_{1}\left\langle|\theta|^{2}, \varphi_{T}(t)\right\rangle .
\end{aligned}
$$

Здесь $\mu_{1}>0$ - первое собственное число оператора Лапласа в области $\omega$. 
Подставив эти оценки в формулу (1.12) и зафиксировав $\alpha>0$ столь мальм, чтобы

получим:

$$
C_{2} \alpha+C_{1} \alpha^{2} \leqslant \frac{\varepsilon}{2} \mu_{1}
$$

$$
\left\langle\left|\partial_{t} \theta\right|^{2}, \varphi_{T}(t)\right\rangle+\left\langle|\nabla \theta|^{2}, \varphi_{T}(t)\right\rangle \leqslant C\left\langle\left|g_{1}\right|^{2}, \varphi_{T}(t)\right\rangle .
$$

Из определения функции $\varphi_{T}(t)$ следует, что $\varphi_{T}(t) \geqslant e^{-\alpha}$ при $t \in[T, T+1]$. Таким образом,

$$
\left\|\theta, \Omega_{T}\right\|_{1,2}^{2} \leqslant e^{\alpha}\left(\left\langle\left|\partial_{t} \theta\right|^{2}, \varphi_{T}(t)\right\rangle+\left\langle|\nabla \theta|^{2}, \varphi_{T}(t)\right\rangle\right) \leqslant C_{1}\left\langle\left|g_{1}\right|^{2}, \varphi_{T}(t)\right\rangle .
$$

Используя условия (1.10), получим:

$$
\left\langle\left|g_{1}\right|^{2}, \varphi_{T}(t)\right\rangle \leqslant 2\left\langle|g|^{2}, e^{-2 \lambda_{0} t} \varphi_{T}(t)\right\rangle+C e^{-\alpha T}\left\|u_{0}\right\|_{V_{0}}^{2}
$$

Подставив эту оценку в правую часть неравенства (1.18) и вспомнив, что $\theta(t)=$ $u(t) e^{-\lambda_{0} t}-v(t)$, получим оценку (1.9). Лемма 1.3 доказана.

Лемма 1.4. Пусть и - решение уравнения (1.1). Тогда для любого $1<r \leqslant p$ и любого $\nu>0$ справедлива следующая оценка:

$$
\left\|u, \Omega_{T}\right\|_{2, r} \leqslant C_{\nu}\left(\left\|u, \Omega_{T-\nu, T+1+\nu}\right\|_{1, r}+\left\|g, \Omega_{T-\nu, T+1+\nu}\right\|_{0, r}+\chi(\nu-T)\left\|u_{0}\right\|_{V_{0}}\right) .
$$

Здесь $\Omega_{T_{1}, T_{2}}=\left[T_{1}, T_{2}\right] \times \omega$ при $T_{2}>T_{1} \geqslant 0 u \Omega_{T_{1}, T_{2}}=\left[0, T_{2}\right] \times \omega$ nрu $T_{1}<0 \leqslant T_{2}$, $\chi(z)$ - функция Хевисайда, равная нулю при $z<0$ и единице при $z \geqslant 0$, и константа $C_{\nu}$ не зависит от $T$.

ДоказАтЕльство. Пусть $\psi_{T}(t) \in C_{0}^{\infty}\left(\mathbb{R}_{+}\right)-$срезающая функция, $\psi_{T}(t)=1$ при $t \in[T, T+1]$ и $\psi_{T}(t)=0$ при $t \notin[T-\nu, T+1+\nu]$. Рассмотрим функцию $w=\psi_{T}(t) u(t)$. Из уравнения (1.1) следует:

$$
\left\{\begin{array}{l}
\partial_{t}^{2} w+\Delta_{x} w=h_{u}(t) \\
\left.w\right|_{\partial \omega}=0,\left.\quad w\right|_{t=T+1+\nu}=0 \\
\left.w\right|_{t=\max \{T-\nu, 0\}}=\psi_{T}(0) u_{0}
\end{array}\right.
$$

Здесь

$$
h_{u}=\psi_{T}^{\prime \prime} u+2 \psi_{T}^{\prime} \partial_{t} u+a^{-1} \psi_{T}\left(\gamma \partial_{t} u+q(x, t) u+g(t)\right) .
$$

Согласно формуле (1.21)

$$
\left\|h_{u}, \Omega_{T-\nu, T+1+\nu}\right\|_{0, r} \leqslant C_{\nu}\left(\left\|u, \Omega_{T-\nu, T+1+\nu}\right\|_{1, r}+\left\|g, \Omega_{T-\nu, T+1+\nu}\right\|_{0, r}\right) .
$$

Используя теорему об $\left(W_{2, r}, L_{r}\right)$-регулярности решений уравнения Лапласа (учитывая, что $\psi_{T}(0)=0$ при $\left.T \geqslant \nu\right)$, получим:

$$
\begin{aligned}
\left\|u, \Omega_{T}\right\|_{2, r} & \leqslant\left\|w, \Omega_{T-\nu, T+1+\nu}\right\|_{2, r} \\
& \leqslant C_{1}\left(\left\|h_{u}, \Omega_{T-\nu, T+1+\nu}\right\|_{0, r}+\chi(\nu-T)\left\|u_{0}\right\|_{V_{0}}\right) .
\end{aligned}
$$

Оценка (1.19) следует непосредственно из неравенств (1.22) и (1.23). Лемма 1.4 доказана. 
ЛЕмма 1.5. Пусть и-решение уравнения (1.1). Тогда

$$
\left\|u, \Omega_{T}\right\|_{2, p} \leqslant C\left(\left\|u, \Omega_{T-1, T+2}\right\|_{1,2}+\left\|g, \Omega_{T-1, T+2}\right\|_{0, p}+\chi(1-T)\left\|u_{0}\right\|_{V_{0}}\right) .
$$

ДокАЗАТЕЛьство. Согласно теореме вложения С. Л. Соболева

$$
\left\|u, \Omega_{T-\nu, T+1+\nu}\right\|_{1, l(r)} \leqslant C\left\|u, \Omega_{T-\nu, T+1+\nu}\right\|_{2, r} .
$$

Здесь

$$
l(r)= \begin{cases}r \frac{n+1}{n+1-r} & \text { при } r<n+1 \\ \infty & \text { при } r>n+1\end{cases}
$$

Из оценок (1.19) и (1.25) получаем:

$\left\|u, \Omega_{T}\right\|_{1, m(r)} \leqslant C\left(\left\|u, \Omega_{T-\nu, T+1+\nu}\right\|_{1, r}+\left\|g, \Omega_{T-\nu, T+1+\nu}\right\|_{0, r}+\chi(\nu-T)\left\|u_{0}\right\|_{V_{0}}\right)$.

Здесь $m(r)=\min \{l(r), p\}$. Определим последовательность показателей $r_{i}$ следующим образом: $r_{0}=2, r_{N+1}=m\left(r_{N}\right)$. Из формулы $(1.26)$ следует, что $r_{N}=p$ при $N \geqslant N_{0}=\left[\frac{\ln p / 2}{\ln (1+2 /(n+1))}\right]+1$, где символом $[z]$ обозначена целая часть числа $z$.

Итерируя формулу (1.27) $N_{0}$ раз, начав с $r=2$, получим:

$$
\begin{aligned}
\left\|u, \Omega_{T}\right\|_{1, p} \leqslant C\left(\left\|u, \Omega_{T-N_{0} \nu, T+1+N_{0} \nu}\right\|_{1,2}\right. & \\
& \left.+\left\|g, \Omega_{T-N_{0} \nu, T+1+N_{0} \nu}\right\|_{0, p}+\chi\left(N_{0} \nu-T\right)\left\|u_{0}\right\|_{V_{0}}\right) .
\end{aligned}
$$

Подставив эту оценку в формулу (1.19) и выбрав $\nu=\frac{1}{N_{0}+1}$, получим оценку (1.24). Лемма 1.5 доказана.

Подставив оценку (1.9) в неравенство (1.24), получим оценку (1.8).

Единственность решения задачи (1.1) следует из этой оценки и линейности системы (1.1). Таким образом, для завершения доказательства теоремы 1.1 осталось доказать существование решения задачи (1.1). Для построения этого решения нам понадобится аналог теоремы 1.1 для вспомогательной задачи вида (1.1) в конечном цилиндре $\Omega_{0, M}, M \in \mathbb{N}$,

$$
\left\{\begin{array}{l}
a\left(\Delta_{x} u+\partial_{t}^{2} u\right)-\gamma \partial_{t} u-q(t, x) u=g(t) \\
\left.u\right|_{\partial \omega}=0,\left.\quad u\right|_{t=0}=u_{0},\left.\quad u\right|_{t=M}=u_{1}
\end{array}\right.
$$

ЛЕмма 1.6. Задача (1.29) имеет единственное решение для любъх функиий $u_{0}, u_{1} \in V_{0}, 0 \leqslant T \leqslant M-1$, и справедлива оценка

$$
\begin{aligned}
\left\|u, \Omega_{T}\right\|_{2, p}^{p} \leqslant C e^{p \lambda_{0} T}\left(\left\|u_{0}\right\|_{V_{0}}^{p} e^{-\alpha T}+\left\|u_{1}\right\|_{V_{0}}^{p} e^{-\alpha(M-T)-p \lambda_{0} M}\right. \\
\left.+\int_{0}^{M} e^{-\alpha|T-s|-p \lambda_{0} s}\|g(s)\|_{0, p}^{p} d s\right) .
\end{aligned}
$$

Более того, константа $C$ в последнем неравенстве не зависит от $M \in \mathbb{N}$. 
ДокАЗАТЕЛЬСТво. Вывод априорной оценки (1.30) в случае ограниченного цилиндра $\Omega_{0, M}$ совершенно аналогичен доказательству оценки (1.8) в случае неограниченного цилиндра $\Omega_{+}$, проведенному вьше (леммы $1.3-1.5$ ), и поэтому здесь не приводится. Существование решения задачи (1.29) доказывается на основе априорной оценки (1.30) при помоши принципа Лере-Шаудера (см., например, [4]). Лемма 1.6 доказана.

Завершим доказательство теоремы 1.1. Рассмотрим последовательность $u_{M}$ решений задач (1.29) в цилиндрах $\Omega_{0, M}, M \in \mathbb{N}$, с условиями $\left.u_{M}\right|_{t=0}=u_{0}$, $\left.u_{M}\right|_{t=M}=0$. Согласно равномерной оценке (1.30) последовательность $u_{M}$ $(M \geqslant T)$ ограничена в пространстве $W_{2, p}\left(\Omega_{T}\right)$ для любого $T \geqslant 0$. Используя рефилексивность пространства $W_{2, p}$ и канторовскую диагональную процедуру, выделим из $u_{M}$ подпоследовательность $u_{M_{k}}$, слабо сходящуюся к некоторой функции $u$ в пространстве $W_{2, p}\left(\Omega_{T}\right)$ для любого $T \in \mathbb{N}$. Нетрудно проверить (см. [4]), что $u$ - искомое решение задачи (1.1). Теорема 1.1 доказана.

В дальнейшем нам понадобится оценка снизу норм решений задачи (1.1).

ТЕОРема 1.2. Пусть функиия $u \in W_{2,2}\left(\Omega_{T}\right)$ для любого $T \geqslant 0$ и удовлетворяет уравнению (1.1) с $g \equiv 0$. Тогда справедлива оченка

$$
\|u(t)\|_{1,2}^{2}+\left\|\partial_{t} u(t)\right\|_{0,2}^{2} \geqslant C\left(\|u(0)\|_{1,2}^{2}+\left\|\partial_{t} u(0)\right\|_{0,2}^{2}\right) e^{-\beta t^{2}+C_{u} t}
$$

Здесь константа $C_{u} \in \mathbb{R}$ зависит, вообще говоря, от функции и, а константы $\beta, C \in \mathbb{R}_{+}$зависят лишь от коэффициентов уравнения (1.1).

ДокАЗАТЕЛЬСТво этой теоремы при немного более сильных предположениях относительно функции $u$ приведено в работе [14]. Для удобства читателя мы приводим ниже схему этого доказательства с необходимыми в нашем случае изменениями.

Введем функции

$$
p(t)=\partial_{t} u(t)+\left(-\Delta_{x}\right)^{1 / 2} u(t) \text { и } r(t)=\partial_{t} u(t)-\left(-\Delta_{x}\right)^{1 / 2} u(t) .
$$

Тогда, так как $u \in W_{2,2}\left(\Omega_{T}\right)$ при любом $T>0$, то для любого $M>0$

$$
p, r \in W_{1,2}\left([0, M], L_{2}(\omega)\right) \cap L_{2}\left([0, M], W_{1,2}^{0}(\omega)\right) \subset C\left([0, M], L_{2}(\omega)\right) .
$$

Так как $u$ удовлетворяет уравнению (1.1), то, как нетрудно проверить,

$$
\begin{aligned}
& \partial_{t} p-\left(-\Delta_{x}\right)^{1 / 2} p=a^{-1} \gamma \frac{p+r}{2}+a^{-1} q\left(-\Delta_{x}\right)^{-1 / 2} \frac{p-r}{2}, \\
& \partial_{t} r+\left(-\Delta_{x}\right)^{1 / 2} r=a^{-1} \gamma \frac{p+r}{2}+a^{-1} q\left(-\Delta_{x}\right)^{-1 / 2} \frac{p-r}{2} .
\end{aligned}
$$

Или, обозначив $\xi_{u}(t)=\left(\begin{array}{c}p(t) \\ r(t)\end{array}\right)$,

$$
\partial_{t} \xi_{u}(t)-\mathscr{B} \xi_{u}(t)=\mathscr{T}(t) \xi_{u}(t), \quad \text { где } \mathscr{B}=\left(\begin{array}{cc}
-\left(-\Delta_{x}\right)^{1 / 2} & 0 \\
0 & \left(-\Delta_{x}\right)^{1 / 2}
\end{array}\right),
$$


а оператор $\mathscr{T}(t)$ удовлетворяет равномерной по $t \in[0, \infty]$ оценке

$$
\left\|\mathscr{T}(t) \xi_{u}\right\|_{0,2} \leqslant C\left\|\xi_{u}\right\|_{0,2}
$$

Пусть $y(t)=\left\|\xi_{u}(t)\right\|_{0,2}^{2}$. Предположим, что $y(0)>0$ (в противном случае доказывать нечего). Тогда согласно (1.33) $y(t)>0$ при $t \in[0, M]$ для некоторого $M>0$.

Рассмотрим функцию

$$
l(t)=\ln y(t)-\int_{0}^{t} F(s) d s, \text { где } t \in[0, M], \text { а } F(t)=\frac{2\left(\mathscr{T}(t) \xi_{u}(t), \xi_{u}(t)\right)}{y(t)} .
$$

Для завершения доказательства теоремы 1.2 понадобится следующая лемма.

ЛЕмма 1.7. Пусть $и(t)$ удовлетворяет уравнению $(1.1)$, а функиия $l(t)$ определена формулой (1.35) Тогда $l \in A C([0, M]), l^{\prime} \in A C([0, M])$ и справедлива оценка

$$
l^{\prime \prime}(t)+4 C^{2} \geqslant 0
$$

Здесь константа $C$ такая же, как и в формуле (1.34).

ДокАЗАТЕЛьство. Вывод оценки (1.36) приведен в [14]. Абсолютная непрерывность функции $l(t)$ следует из (1.33). Абсолютная непрерывность производной $l^{\prime}(t)$ следует из (1.33) и формулы

$$
l^{\prime}(t)=\frac{2\left(\mathscr{B} \xi_{u}(t), \xi_{u}(t)\right)}{y(t)}
$$

которая проверяется непосредственным вычислением. Лемма 1.7 доказана.

Завершим доказательство теоремы 1.2. Интегрируя дважды оценку (1.36), получим:

$$
y(t) \geqslant y(0) e^{-2 C^{2} t^{2}+l^{\prime}(0) t+\int_{0}^{t} F(s) d s} .
$$

Из неравенства (1.34) следует, что $\int_{0}^{t} F(s) d s \geqslant-4 C t$. Таким образом,

$$
\|p(t)\|_{0,2}^{2}+\|r(t)\|_{0,2}^{2} \geqslant\left(\|p(0)\|_{0,2}^{2}+\|r(0)\|_{0,2}^{2}\right) e^{-2 C^{2} t^{2}-4 C t+l^{\prime}(0) t} .
$$

Теорема 1.2 доказана.

СлЕДСТВИЕ 1.1. Пусть $u_{1}(t)$ и $u_{2}(t)$ - два решения уравнения (1.1). Тогда если для некоторого $T \geqslant 0 u_{1}(T)=u_{2}(T)$, то $u_{1} \equiv u_{2}$.

Действительно, $u(t)=u_{1}(t)-u_{2}(t)=0$ для любого $t \geqslant T$ согласно теореме 1.1 , следовательно, $\partial_{t} u(T)=0$. Из оценки (1.31) получаем теперь, что $u(0)=0$, a значит, $u(t)=0$ для любого $t \in \mathbb{R}_{+}$. 


\section{§ 2. Нелинейная система. Априорные оценки. Существование решений}

В этом параграфе мы выведем априорную оценку (0.9) и на ее основе покажем, что для любого $u_{0} \in V_{0}$ задача $(0.1)$ имеет хотя бы одно решение. Предполагается, что $а$ и $\gamma$ - самосопряженные матрицы $\left(a, \gamma \in \mathscr{L}\left(\mathbb{R}^{k}, \mathbb{R}^{k}\right)\right)$, причем $a>0$, а нелинейная функция $f \in C\left(\mathbb{R}^{k}, \mathbb{R}^{k}\right)$ и удовлетворяет п. 2 условий $(0.3)$.

В отличие от предыдушего параграфа здесь мы будем рассматривать лишш ограниченные при $t \rightarrow \infty$ решения уравнения (0.1), т.е. решения $u(t)$, имеющие конечную норму (0.5). При этом предполагается, что $g$ удовлетворяет условию (0.4) и $u_{0} \in V_{0}$.

Основным результатом этого параграфа является следующая теорема.

ТЕОРЕМа 2.1. Пусть выполнены перечисленные выше условия и пусть и решение задачи (0.1). Тогда справедлива оценка (0.9).

Для доказательства этой теоремы нам понадобится ряд вспомогательных утверждений.

ЛЕмма 2.1. Пусть и-решение задачи (0.1). Тогда

$$
\|u(T)\|_{0,2}^{2} \leqslant C\left(\|u(0)\|_{0,2}^{2} e^{-\alpha T}+1+|g|_{b}^{2}\right)
$$

для некоторой положстельной константь $\alpha$.

Доказательство этой оценки также будет проведено в несколько шагов. На первом шаге будет получена грубая оценка вида (2.1), которая в дальнейшем будет уточняться.

ПРЕДЛОЖЕНИЕ 2.1. Пусть $и-$ - ешение задачи (0.1). Тогда

$$
\|u(T)\|_{0,2}^{2} \leqslant C e^{m T}\left(1+|g|_{b}^{2}+\left\|u_{0}\right\|_{0,2}^{2}\right)
$$

для некоторой положительной константы $\mathrm{m}$.

ДокАЗАтЕльство. Умножим скалярно в пространстве $H$ уравнение $(0.1)$ на функцию $u e^{-\varepsilon t} \chi_{\tau}(t)$, где $\chi_{\tau}(t)=\chi(t-\tau)(\chi(z)-$ функция Хевисайда), a $\varepsilon>0$ - достаточно малое положительное число, которое будет определено позднее, и проинтегрируем полученное соотношение по $t \in \mathbb{R}_{+}$

$$
\begin{aligned}
\left\langle a \partial_{t}^{2} u, u \chi_{\tau} e^{-\varepsilon t}\right\rangle & -\left\langle\gamma \partial_{t} u, u \chi_{\tau} e^{-\varepsilon t}\right\rangle \\
& -\left\langle a \nabla_{x} u, \nabla_{x} u \chi_{\tau} e^{-\varepsilon t}\right\rangle-\left\langle f(u), u \chi_{\tau} e^{-\varepsilon t}\right\rangle=\left\langle g, u \chi_{\tau} e^{-\varepsilon t}\right\rangle
\end{aligned}
$$

Преобразуем первое и второе слагаемое формулы (2.3) при помощи интегрирования по частям

$$
\begin{aligned}
-\left\langle a \partial_{t}^{2} u, u \chi_{\tau} e^{-\varepsilon t}\right\rangle & =\left\langle a \partial_{t} u, \partial_{t} u \chi_{\tau} e^{-\varepsilon t}\right\rangle-\varepsilon\left\langle a \partial_{t} u, u \chi_{\tau} e^{-\varepsilon t}\right\rangle+\left(a \partial_{t} u(\tau), u(\tau)\right) e^{-\varepsilon \tau} \\
& \geqslant C\left\langle\left|\partial_{t} u\right|^{2}, \chi_{\tau} e^{-\varepsilon t}\right\rangle-C_{1} \varepsilon^{2}\left\langle|u|^{2}, \chi_{\tau} e^{-\varepsilon t}\right\rangle+y^{\prime}(\tau) e^{-\varepsilon \tau}
\end{aligned}
$$


Здесь и далее $y(\tau)=\frac{1}{2}(a u(\tau), u(\tau))$. Имеем:

$$
\begin{aligned}
-\left\langle\gamma \partial_{t} u, u \chi_{\tau} e^{-\varepsilon t}\right\rangle & =-\frac{1}{2} \varepsilon\left\langle\gamma u, u \chi_{\tau} e^{-\varepsilon t}\right\rangle+\frac{1}{2}(\gamma u(\tau), u(\tau)) e^{-\varepsilon \tau} \\
& \leqslant C_{2} \varepsilon\left\langle|u|^{2}, \chi_{\tau} e^{-\varepsilon t}\right\rangle+C_{3} y(\tau) e^{-\varepsilon \tau}
\end{aligned}
$$

Согласно п. 2 условий (0.3)

$$
-\left\langle f(u), u \chi_{\tau} e^{-\varepsilon t}\right\rangle \leqslant\left\langle C, \chi_{\tau} e^{-\varepsilon t}\right\rangle \leqslant C_{1} .
$$

Согласно неравенству Фридрихса

$$
\left\langle a \nabla_{x} u, \nabla_{x} u \chi_{\tau} e^{-\varepsilon t}\right\rangle \geqslant 2 C_{4}\left\langle|u|^{2}, \chi_{\tau} e^{-\varepsilon t}\right\rangle .
$$

Оценив последнее слагаемое в формуле (2.3) при помощи неравенства Гёльдера, получим:

$$
\begin{aligned}
\left|\left\langle g, u \chi_{\tau} e^{-\varepsilon t}\right\rangle\right| & \leqslant C_{4}\left\langle|u|^{2}, \chi_{\tau} e^{-\varepsilon t}\right\rangle+C_{5}\left\langle|g|^{2}, \chi_{\tau} e^{-\varepsilon t}\right\rangle \\
& \leqslant C_{4}\left\langle|u|^{2}, \chi_{\tau} e^{-\varepsilon t}\right\rangle+C_{6}|g|_{b}^{2} .
\end{aligned}
$$

Подставив эти оценки в формулу (2.3) и выбрав $\varepsilon>0$ так, чтобы $C_{1} \varepsilon^{2}+C_{2} \varepsilon \leqslant C_{3}$, получим:

$$
\left(y^{\prime}(\tau)-C_{3} y(\tau)\right) e^{-\varepsilon \tau} \leqslant C\left(1+|g|_{b}^{2}\right) .
$$

Применив неравенство Гронуолла к оценке (2.8), получим оценку (2.2). Предложение 2.1 доказано.

ПРЕДЛОЖЕНИЕ 2.2. Пусть и - решение уравнения (0.1). Тогда для $T \geqslant 1$ справедлива следующая оценка:

$$
\left\|u, \Omega_{T}\right\|_{1,2}^{2} \leqslant C\left(\left\|u, \Omega_{0}\right\|_{0,2}^{2} e^{-\alpha T}+1+|g|_{b}^{2}\right)
$$

ДоказАТЕльство. Введем еше одну срезаюшую функцию $\psi(t) \in C_{0}^{\infty}\left(\mathbb{R}_{+}\right)$ такую, что $\psi(t) \geqslant 0, \psi(t)=0$ при $t \leqslant 0$ и $\psi(t)=1$ при $t \geqslant 1$. Умножим аналогично доказательству леммы 1.3 уравнение (0.1) скалярно в пространстве $L_{2}\left(\Omega_{+}\right)$на функцию $u(t) \psi(t) e^{-\alpha|T-t|}=u \psi \varphi_{T}$. Получим:

$$
\begin{aligned}
\left\langle a \partial_{t}^{2} u, u \psi \varphi_{T}\right\rangle-\langle a \nabla u, \nabla & \left.\left(u \psi \varphi_{T}\right)\right\rangle \\
& -\left\langle\gamma \partial_{t} u, u \psi \varphi_{T}\right\rangle-\left\langle f(u), u \psi \varphi_{T}\right\rangle=\left\langle g, u \psi \varphi_{T}\right\rangle .
\end{aligned}
$$

Оценим отдельно каждое слагаемое в формуле (2.10). Интегрируя по частям и используя тот факт, что supp $\psi^{\prime} \subset[0,1]$, получим аналогично (1.14)

$$
\begin{aligned}
-\left\langle a \partial_{t}^{2} u, u \psi \varphi_{T}\right\rangle & =\left\langle a \partial_{t} u . \partial_{t} u, \psi \varphi_{T}\right\rangle+\frac{1}{2}\left\langle\partial_{t}[a u . u], \psi^{\prime} \varphi_{T}\right\rangle+\left\langle a \partial_{t} u, u \psi \varphi_{T}^{\prime}\right\rangle \\
& =\left\langle a \partial_{t} u . \partial_{t} u, \psi \varphi_{T}\right\rangle+\left\langle a \partial_{t} u, u \psi \varphi_{T}^{\prime}\right\rangle-\frac{1}{2}\left\langle a u . u,\left(\psi^{\prime} \varphi_{T}\right)^{\prime}\right\rangle \\
& \geqslant C\left\langle\left|\partial_{t} u\right|^{2}, \psi \varphi_{T}\right\rangle-C_{1} \alpha^{2}\left\langle|u|^{2}, \psi \varphi_{T}\right\rangle-C_{2}\left\|u, \Omega_{0}\right\|_{0,2}^{2} e^{-\alpha T}
\end{aligned}
$$


Используя самосопряженность матрицы $\gamma$, получим аналогично (1.15)

$$
\begin{aligned}
\left\langle\gamma \partial_{t} u, u \psi \varphi_{T}\right\rangle & =-\frac{1}{2}\left\langle\gamma u . u, \psi \varphi_{T}^{\prime}\right\rangle-\frac{1}{2}\left\langle\gamma u . u, \psi^{\prime} \varphi_{T}\right\rangle \\
& \geqslant-C\left(\alpha\left\langle|u|^{2}, \psi \varphi_{T}\right\rangle+\left\|u, \Omega_{0}\right\|_{0,2}^{2} e^{-\alpha T}\right) .
\end{aligned}
$$

Согласно п. 2 условий $(0.3)$

$$
\left\langle f(u), u \psi \varphi_{T}\right\rangle=\left\langle f(u) . u, \psi \varphi_{T}\right\rangle \geqslant-\left\langle C, \psi \varphi_{T}\right\rangle \geqslant-C_{1} .
$$

Согласно неравенству Гёльдера

$$
\left|\left\langle g, u \psi \varphi_{T}\right\rangle\right| \leqslant \mu\left\langle|u|^{2}, \psi \varphi_{T}\right\rangle+C_{\mu}\left\langle|g|^{2}, \psi \varphi_{T}\right\rangle
$$

Подставив оценки (2.11)-(2.14) в уравнение (2.10), получим

$$
\left\langle\left|\partial_{t} u\right|^{2}+|\nabla u|^{2}, \psi \varphi_{T}\right\rangle \leqslant C\left(\left\|u, \Omega_{0}\right\|_{0,2}^{2} e^{-\alpha T}+1+\left\langle|g|^{2}, \varphi_{T}\right\rangle\right) .
$$

Оценка (2.9) следует из оценки (2.15) так же, как и при доказательстве леммы 1.3. Предложение 2.2 доказано.

ДокАЗАТЕЛЬСтво ЛЕммы 2.1. Подставив оценку (2.2) в оценку (2.9) и использовав очевидную оценку

$$
\|u(T)\|_{0,2} \leqslant C\left\|u, \Omega_{T}\right\|_{1,2},
$$

получаем неравенство $(2.1)$ в случае $T \geqslant 1$. В случае $T \leqslant 1$ неравенство $(2.1)$ немедленно следует из оценки (2.2). Лемма 2.1 доказана.

Лемма 2.2. Пусть и-решение задачи (0.1). Тогда

$$
\|u(T)\|_{0, \infty} \leqslant C\left(\left\|u_{0}\right\|_{V_{0}} e^{-\alpha T}+1+|g|_{b}\right) .
$$

ДокаЗАТЕЛЬСтво. Рассмотрим функцию $w(t, x)=a u(t, x) \cdot u(t, x)$. Нетрудно проверить, что эта функция удовлетворяет уравнению

$$
\left\{\begin{array}{l}
\partial_{t}^{2} w(t)+\Delta_{x} w(t)=h_{u}(t), \\
\left.w\right|_{t=0}=a u_{0} \cdot u_{0}
\end{array}\right.
$$

Здесь

$$
\begin{aligned}
h_{u}(t)= & 2\left(a \partial_{t} u(t) \cdot \partial_{t} u(t)+a \nabla u(t) \cdot \nabla u(t)+\gamma \partial_{t} u(t) \cdot u(t)\right. \\
& +f(u(t)) \cdot u(t)+g(t) \cdot u(t)) \\
\geqslant & -C_{1}\left(1+|g(t)| \cdot|u(t)|+|u(t)|^{2}\right) \equiv h(t) .
\end{aligned}
$$


Рассмотрим вспомогательную задачу

$$
\left\{\begin{array}{l}
\partial_{t}^{2} v(t)+\Delta_{x} v(t)=h(t), \\
\left.v\right|_{t=0}=a u_{0} . u_{0} .
\end{array}\right.
$$

Согласно теореме вложения С. Л. Соболева $W_{2, p} \subset C$ при $p>(n+1) / 2$. Таким образом, из условия (0.5) следует, что $h \in L_{p}\left(\Omega_{T}\right)$ при любом $T \geqslant 0$ и $|h|_{b}<\infty$.

Согласно теореме $1.1 \mathrm{c} \gamma=0, q=0, K=0, \lambda_{0}=0$

$$
\left\|v, \Omega_{T}\right\|_{2, p}^{p} \leqslant C\left(\left\|u_{0}\right\|_{V_{0}}^{2 p} e^{-\alpha T}+\int_{\mathbb{R}_{+}} e^{-\alpha|T-s|}\|h(s)\|_{0, p}^{p} d s\right) .
$$

Согласно принципу максимума для ограниченных решений уравнения (2.19) (см. [4])

$$
w(t, x) \leqslant v(t, x) \text { почти всюду в } \Omega_{+} .
$$

Так как $p>(n+1) / 2$, то из $(2.20)$ и $(2.21)$, учитьвая положительность функции $w$, получаем:

$$
\|u(T)\|_{0, \infty}^{2 p} \leqslant C\left(\left\|u_{0}\right\|_{V_{0}}^{2 p} e^{-\alpha T}+\int_{\mathbb{R}_{+}} e^{-\alpha|T-s|}\|h(s)\|_{0, p}^{p} d s\right) .
$$

Оценим функцию $h$ при помощи неравенства Гёльдера

$$
\begin{aligned}
\|h(s)\|_{0, p}^{p} & \leqslant C\left(1+\left(|g(s)|^{p}|u(s)|^{p}, 1\right)+\|u(s)\|_{0,2 p}^{2 p}\right) \\
& \leqslant C_{1}\left(1+\|g(s)\|_{0, p}^{p}\|u(s)\|_{0, \infty}^{p}+\|u(s)\|_{0,2 p}^{2 p}\right) .
\end{aligned}
$$

Оценим последнее слагаемое в правой части формулы (2.23) следуюшим образом:

$$
\|u(s)\|_{0,2 p}^{2 p} \leqslant\|u(s)\|_{0, \infty}^{2 p(1-\theta)}\|u(s)\|_{0,2}^{2 p \theta} \leqslant C_{\mu}\|u(s)\|_{0,2}^{2 p}+\mu\|u(s)\|_{0, \infty}^{2 p} .
$$

Здесь $\theta=1 / p \in(0,1)$ и оценка $(2.24)$ справедлива при любом $\mu>0$.

Аналогично

$$
\begin{aligned}
& \int_{\mathbb{R}_{+}} e^{-\alpha|T-s|}\|g(s)\|_{0, p}^{p}\|u(s)\|_{0, \infty}^{p} d s \\
& \leqslant C|g|_{b}^{p} \sup _{s \geqslant 0}\left\{e^{-\alpha|T-s| / 2}\|u(s)\|_{0, \infty}^{p}\right\} \\
& \leqslant C_{\mu}|g|_{b}^{2 p}+\mu \sup _{s \geqslant 0}\left\{e^{-\alpha|T-s|}\|u(s)\|_{0, \infty}^{2 p}\right\} .
\end{aligned}
$$

Подставляя оценки $(2.23),(2.24)$ и $(2.25)$ в неравенство $(2.22)$ и используя оценку $L_{2}$-нормы функции $u(s)$, доказанную в лемме 2.1 , получим после несложных преобразований

$$
\|u(T)\|_{0, \infty}^{2 p} \leqslant C_{\mu}\left(1+|g|_{b}^{2 p}+\left\|u_{0}\right\|_{V_{0}}^{2 p} e^{-\alpha T}\right)+\mu \sup _{s \geqslant 0}\left\{e^{-\alpha|T-s|}\|u(s)\|_{0, \infty}^{2 p}\right\} .
$$

Для завершения доказательства леммы 2.2 нам понадобится следуюшее предложение. 
ПРЕДЛОЖЕНИЕ 2.3. Пусть функция $z \in C_{b}\left(\mathbb{R}_{+}\right)$такова, что выполнено следующее неравенство

$$
z(t) \leqslant C_{1}+C_{0} e^{-\alpha t}+\mu \sup _{s \geqslant 0}\left\{e^{-\beta|t-s|} z(s)\right\}
$$

при некоторых $\alpha \geqslant \beta>0$ и $\mu<1 / 2$. Тогда

$$
z(t) \leqslant 2\left(C_{0} e^{-\beta t}+C_{1}\right) .
$$

ДокАЗАТЕЛЬСТво. Умножим неравенство $(2.27)$ на $e^{-\beta|l-t|}, l \in \mathbb{R}_{+}$, и возьмем $\sup _{t \geqslant 0}$ от обеих частей полученного неравенства. Получим:

$$
\begin{aligned}
\sup _{t \geqslant 0} e^{-\beta|l-t|} z(t) \leqslant C_{0} \sup _{t \geqslant 0} e^{-\beta|l-t|} e^{-\alpha t}+C_{1} \sup _{t \geqslant 0} e^{-\beta|l-t|} & \\
& +\mu \sup _{t \geqslant 0} \sup _{s \geqslant 0}\left\{e^{-\beta(|t-s|+|t-l|)} z(s)\right\} .
\end{aligned}
$$

Имеем: $\sup _{t \geqslant 0} e^{-\beta|l-t|-\alpha t}=e^{-\beta l}$. Изменяя порядок предельных переходов в последнем слагаемом в правой части неравенства (2.29), получим:

$$
\begin{aligned}
\sup _{t \geqslant 0} \sup _{s \geqslant 0}\left\{e^{-\beta(|t-s|+|t-l|)} z(s)\right\} & =\sup _{s \geqslant 0}\left\{\sup _{t \geqslant 0} e^{-\beta(|t-s|+|t-l|)} z(s)\right\} \\
& =\sup _{s \geqslant 0} e^{-\beta|l-s|} z(s)=\sup _{t \geqslant 0}\left\{e^{-\beta|l-t|} z(t)\right\} .
\end{aligned}
$$

Подставив эти формулы в неравенство (2.29) и использовав, что $\mu \leqslant 1 / 2$, будем иметь

$$
\sup _{t \geqslant 0} e^{-\beta|l-t|} z(t) \leqslant 2\left(C_{0} e^{-\beta l}+C_{1}\right)
$$

Подставив эту оценку в неравенство (2.27), получим оценку (2.28). Предложение 2.3 доказано.

Завершим доказательство леммы 2.2. Применим предложение 2.3 к неравенству $(2.26)$ и $z(t)=\|u(t)\|_{0, \infty}^{2 p}$. Оценка (2.16) немедленно следует теперь из неравенства (2.28). Лемма 2.2 доказана.

Лемма 2.3. Пусть и-решение уравнения (0.1). Тогда

$$
\left\|f(u), \Omega_{T}\right\|_{0, \infty} \leqslant Q\left(\left\|u_{0}\right\|_{V_{0}}\right) e^{-\alpha T}+Q\left(|g|_{b}\right)
$$

для некоторой монотонной функции $Q$.

Вывод оценки (2.30) из оценки (2.16) приведен в [4].

Завершим доказательство теоремы 2.1. Запишем уравнение $(0.1)$ в виде линейного уравнения

$$
a \partial_{t}^{2} u-\gamma \partial_{t} u+a \Delta_{x} u=g(t)+f(u(t))=h(t) .
$$


Уравнение (2.31) имеет вид (1.1) при $q(t, x) \equiv 0$. Кроме того, очевидно, что пучок $(0.7)$ с $K=0$ удовлетворяет условиям $(0.8)$ при $\lambda_{0}=0$. Применив к уравнению (2.31) оценку (1.8) с $\lambda_{0}=0$, получим:

$$
\left\|u, \Omega_{T}\right\|_{2, p}^{p} \leqslant C\left\|u_{0}\right\|_{V_{0}}^{p} e^{-\alpha T}+C \int_{\mathbb{R}_{+}} e^{-\alpha|T-s|}\|h(s)\|_{0, p}^{p} d s
$$

Подставив оценку (2.30) в неравенство (2.32), получим оценку (0.9). Теорема 2.1 доказана.

Для доказательства разрешимости задачи $(0.1)$ нам, как и в $\S 1$, понадобится вспомогательная задача вида (0.1) в конечном цилиндре $\Omega_{0, M}, M \in \mathbb{N}$,

$$
\left\{\begin{array}{l}
a\left(\partial_{t}^{2} u+\Delta_{x} u\right)-\gamma \partial_{t} u-f(u)=g(t), \\
\left.u\right|_{t=0}=u_{0},\left.\quad u\right|_{t=M}=u_{1},\left.\quad u\right|_{\partial \omega}=0 .
\end{array}\right.
$$

Теорема 2.2. Пусть $u_{0}, u_{1} \in V_{0} u и$-решение задачи (2.33). Тогда справедлива следующая оченка:

$$
\left\|u, \Omega_{T}\right\|_{2, p} \leqslant Q\left(\left\|u_{0}\right\|_{V_{0}}^{2}+\left\|u_{1}\right\|_{V_{0}}^{2}\right)\left(e^{-\alpha T}+e^{-\alpha(M-T)}\right)+Q\left(\|g\|_{b}\right) .
$$

Здесь $Q$ - некоторая монотонная функция, не зависящая от $M \geqslant 2$.

ДокАЗАТЕЛЬСтво. Вывод оценки (2.34) совершенно аналогичен доказательству оценки (0.9), проведенному выше (леммы 2.1-2.3). Поэтому ниже мы приведем лишь схему доказательства теоремы 2.2 .

Аналогом оценки (2.1) для случая задачи (2.33) будет следующая равномерная по $M \in \mathbb{N}$ оценка.

Лемма 2.4. Пусть и - решение задачи (2.33). Тогда при $T \in[0, M]$

$$
\|u(T)\|_{0,2}^{2} \leqslant C\left(\left(\|u(0)\|_{0,2}^{2}+\|u(M)\|_{0,2}^{2}\right)\left(e^{-\alpha T}+e^{-\alpha(M-T)}\right)+1+|g|_{b}^{2}\right)
$$

Для доказательства леммы 2.4 нам понадобятся следующие предложения.

ПРЕДЛОЖЕНИЕ 2.4. Пусть и - решение задачи (2.33). Тогда равномерно по $M \in \mathbb{N}$ справедлива оценка

$$
\|u(T)\|_{0,2}^{2} \leqslant C\left(\|u(0)\|_{0,2}^{2}+\|u(M)\|_{0,2}^{2}\right) \min \left\{e^{m T}, e^{m(M-T)}\right\}
$$

Здесъ $m$ - некоторое положительное число, $0 \leqslant T \leqslant M-1$. 
ДокАЗАТЕЛЬСтво. Умножив уравнение (2.33) скалярно в $L_{2}(\omega)$ на функцию $u e^{-\varepsilon t}$ и проинтегрировав по отрезку $[\tau, s] \subset[0, M]$, получим аналогично выводу оценки (2.8) следующую равномерную по $M$ оценку:

$$
\left(y^{\prime}(\tau)-C_{1} y(\tau)\right) e^{-\varepsilon \tau}-\left(y^{\prime}(s)+C_{2} y(s)\right) e^{-\varepsilon s} \leqslant C\left(1+|g|_{b}^{2}\right) .
$$

Без ограничения общности можно считать, что $C_{1}>0$ и $C_{2}>0$.


чим после несложных преобразований

$$
\left(y^{\prime}(\tau)-C_{1} y(\tau)\right) e^{-\varepsilon \tau} \leqslant C\left(1+|g|_{b}^{2}\right)+\left(C_{2}+\varepsilon\right) y(M) e^{C_{2} M}\left(e^{\left(C_{2}+\varepsilon\right) M}-e^{\left(C_{2}+\varepsilon\right) \tau}\right)^{-1} .
$$

Без ограничения общности можно считать также, что $\tau \leqslant M-1$. В этом случае, как нетрудно проверить,

$$
e^{C_{2} M}\left(e^{\left(C_{2}+\varepsilon\right) M}-e^{\left(C_{2}+\varepsilon\right) \tau}\right)^{-1} \leqslant\left(1-e^{-C_{2}-\varepsilon}\right)^{-1} \leqslant C .
$$

Таким образом, из (2.38) следует оценка

$$
\left(y^{\prime}(\tau)-C_{1} y(\tau)\right) e^{-\varepsilon \tau} \leqslant C\left(1+|g|_{b}^{2}+y(M)\right) .
$$

Применив неравенство Гронуолла к оценке (2.39), получим:

$$
y(t) \leqslant C\left(1+|g|_{b}^{2}+|y(0)|+|y(M)|\right) e^{m t} \text { при } t \leqslant M-1
$$

для некоторого $m>0$. Применяя оценку (2.40) к задаче (2.33), в которой $t$ заменено на $M-t$, получим оценку

$$
y(t) \leqslant C\left(1+|g|_{b}^{2}+|y(0)|+|y(M)|\right) e^{m(M-t)} \text { при } t \geqslant 1 .
$$

Неравенство (2.36) следует из оценок (2.40) и (2.41). Предложение 2.4 доказано.

ПРЕДЛОЖЕНИЕ 2.5. Пусть и - решение задачи (2.33). Тогда при T $\in[1, M-2]$ справедлива оценка

$$
\left\|u, \Omega_{T}\right\|_{1,2}^{2} \leqslant C\left(\left\|u, \Omega_{0}\right\|_{0,2}^{2} e^{-\alpha T}+\left\|u, \Omega_{M-1}\right\|_{0,2}^{2} e^{-\alpha(M-T)}+1+|g|_{b}^{2}\right) .
$$

Доказательство предложения 2.5 почти дословно повторяет доказательство предложения 2.2 , только вместо умножения на функцию $u(t) \psi(t) \varphi_{T}(t)$ необходимо умножать уравнение $(2.33)$ на функцию $u(t) \psi(t) \psi(M-t) \varphi_{T}(t)$.

Оценка (2.35) следует из оценок (2.36) и (2.42) (см. доказательство леммы 2.1). Лемма 2.4 доказана. 
ЛЕмма 2.5. Пусть и-решение задачи (2.33). Тогда

$$
\|u(T)\|_{0, \infty}^{2} \leqslant C\left(\left(\left\|u_{0}\right\|_{V_{0}}^{2}+\left\|u_{1}\right\|_{V_{0}}^{2}\right)\left(e^{-\alpha T}+e^{-\alpha(M-T)}\right)+1+|g|_{b}^{2}\right) .
$$

Доказательство леммы 2.5 аналогично доказательству леммы 2.2 , только для оценки $C$-нормы решения вспомогательного уравнения (2.19) нужно воспользоваться оценкой (1.30) вместо оценки (1.8).

Как показано в работе [4], из оценки (2.43) и непрерывности функции $f$ следует оценка

$$
\left\|f(u), \Omega_{T}\right\|_{0, \infty} \leqslant Q\left(\left\|u_{0}\right\|_{V_{0}}^{2}+\left\|u_{1}\right\|_{V_{0}}^{2}\right)\left(e^{-\alpha T}+e^{-\alpha(M-T)}\right)+Q\left(|g|_{b}\right)
$$

для некоторой монотонной функции $Q$.

Записав теперь уравнение (2.33) в виде (2.31) и применив к нему оценку (1.30) с $\lambda_{0}=0$, получим оценку (2.34). Теорема 2.2 доказана.

ТЕОРема 2.3. Задача (0.1) имеет хотя бы одно решение при любом $u_{0} \in V_{0}$.

ДокАЗАТЕЛьство. Как и в линейном случае (см. §1), сначала доказывается разрешимость задачи $(2.33)$ в ограниченном цилиндре $\Omega_{0, M}$. Разрешимость задачи (2.33) выводится на основе априорной оценки (2.34) при помощи принципа Лере-Шаудера (см., например, [4]). Сушествование решения задачи (0.1) доказывается предельным переходом при $M \rightarrow \infty$ (см. доказательство теоремы 1.1). Теорема 2.3 доказана.

ЗАмЕчАниЕ 2.1. Оценка (0.9) получена в работе [4] при несколько более ограничительных условиях на нелинейную функцию $f\left(f(u) . u \geqslant-C_{1}+C_{2}|u|^{2+\varepsilon}\right.$, $\left.C_{2}, \varepsilon>0\right)$, но без требования самосопряженности матрицы $\gamma$.

\section{§ 3. Дифференцируемость решений нелинейного эллиптического уравнения по "начальному" условию $u_{0}$}

В этом параграфе мы докажем однозначную разрешимость краевой задачи (0.1). Кроме того, будет доказана дифференцируемость решений задачи (0.1) по начальному условию $u_{0}$ и проверена эквивалентность задач $(0.1)$ и (0.11).

Всюду в дальнейшем мы вновь предполагаем, что $a$ и $\gamma$-положительно определенные самосопряженные матрицы, нелинейная функция $f$ удовлетворяет условиям (0.3), а операторный пучок (0.7) - условию (0.8) при некотором $\lambda_{0}>0$.

ТЕОРЕМа 3.1. Пусть выполнены перечисленные выше условия. Тогда для любьх двух решений $u_{1}$ и и уравнения (0.1) имеет место следующая оценка:

$$
\left\|u_{1}-u_{2}, \Omega_{T}\right\|_{2, p} \leqslant C\left\|u_{1}(0)-u_{2}(0)\right\|_{V_{0}} e^{\left(\lambda_{0}-\alpha\right) T}
$$

причем константа $C$ в последней формуле зависит от $\left\|u_{1}(0)\right\|_{V_{0}} u\left\|u_{2}(0)\right\|_{V_{0}}$, а константа $\alpha>0$ - только от коэффициентов пучка $L_{K}(\lambda)$. 
ДокаЗАТЕЛЬСТво. Пусть $v=u_{2}-u_{1}$. Тогда, как нетрудно проверить,

где

$$
\left\{\begin{array}{l}
a\left(\partial_{t}^{2} v+\Delta_{x} v\right)-\gamma \partial_{t} v-q_{u_{1}, u_{2}}(t, x) v=0, \\
\left.v\right|_{t=0}=u_{1}(0)-u_{2}(0),
\end{array}\right.
$$

$$
q_{u_{1}, u_{2}}(t, x)=\int_{0}^{1} f^{\prime}\left(u_{1}(t, x)+\rho\left(u_{2}(t, x)-u_{1}(t, x)\right)\right) d \rho .
$$

Из оценки (0.9) и теоремы вложения С. Л. Соболева $W_{2, p} \subset C$ при $p>(n+1) / 2$ получаем:

$$
\left\|u_{i}, \Omega_{+}\right\|_{0, \infty} \leqslant C\left(\left\|u_{i}(0)\right\|_{V_{0}}\right), \quad i=1,2 .
$$

Таким образом, согласно формуле (3.3) и условиям (0.3)

$$
\begin{aligned}
& \text { 1. }\left\|q_{u_{1}, u_{2}}(t, x), \Omega_{+}\right\|_{0, \infty} \leqslant C_{1}\left(\left\|u_{1}(0)\right\|_{V_{0}},\left\|u_{2}(0)\right\|_{V_{0}}\right), \\
& \text { 2. } q_{u_{1}, u_{2}}(t, x) \geqslant-K \text { Id } .
\end{aligned}
$$

Заметим, что по определению решения задачи (0.1) функции $u_{1}(t)$ и $u_{2}(t)$ ограничены при $t \rightarrow \infty$ (т.е. $\left.\left\|u_{i}\right\|_{b}<\infty\right)$, следовательно, функция $v(t)$ также ограничена при $t \rightarrow \infty$, а значит, заведомо

$$
\|v\|_{\lambda_{0}}=\sup _{T \geqslant 0} e^{-\lambda_{0} T}\left\|v, \Omega_{T}\right\|_{2, p}<\infty
$$

Таким образом, для уравнения (3.2) выполнены все условия теоремы 1.1. Оценка (3.1) следует теперь из оценки (1.8). Теорема 3.1 доказана.

СлЕДСТВИЕ 3.1. Из формулы (3.1) следует, что для любого $t \geqslant 0$ корректно определен разрешающий оператор $S_{t}: V_{0} \rightarrow V_{0}$, которьй начальному условию $u_{0} \in V_{0}$ ставит в соответствие решение задачи (0.1) в момент "времени" $t$. Более того, этот оператор является (локально) липшичевым nо $u_{0}$, m.e.

$$
\left\|S_{t}\left(u_{01}\right)-S_{t}\left(u_{02}\right)\right\|_{V_{0}} \leqslant C\left\|u_{01}-u_{02}\right\|_{V_{0}} e^{\left(\lambda_{0}-\alpha\right) t}, \quad u_{01}, u_{02} \in V_{0}
$$

СЛЕДСТВИЕ 3.2. Пусть $V_{0}^{\prime}=W_{1-1 / p, p}^{0}(\omega)$. Тогда из оценки (3.1) следует maкəмe

$$
\left\|\partial_{t} u_{1}(0)-\partial_{t} u_{2}(0)\right\|_{V_{0}^{\prime}} \leqslant C\left\|u_{1}(0)-u_{2}(0)\right\|_{V_{0}} .
$$

Поэтому корректно определен локально липиицев оператор $\Phi: V_{0} \rightarrow V_{0}^{\prime}$, который начальному условию $u_{0} \in V_{0}$ ставит в соответствие производную nо $t$ решения задачи (0.1) в момент времени $t=0$

$$
\left.\partial_{t} u\right|_{t=0}=\Phi\left(\left.u\right|_{t=0}\right)
$$

Рассматривая момент времени $t=\tau>0$ вместо $t=0$, аналогично (3.7) получим соотношение

$$
\left\{\begin{array}{l}
\left.\partial_{t} u\right|_{t=\tau}=\Phi_{\tau}\left(\left.u\right|_{t=\tau}\right) \\
\left.u\right|_{t=0}=u_{0}
\end{array}\right.
$$

справедливое для любого решения и задачи (0.1) и любого $\tau \geqslant 0$ (здесь через $\Phi_{\tau}: V_{0} \rightarrow V_{0}^{\prime}$ обозначен оператор $\Phi$, соответствующий задаче $(0.1)$, в которой $g(t)$ заменено на $g(t+\tau))$. 
СлеДСТВИЕ 3.3. Onератор $S_{t}: V_{0} \rightarrow V_{0}$, определенный в следствии 3.1, является инбективным при любом $t \geqslant 0$, т.е. из $S_{t} \xi_{1}=S_{t} \xi_{2}$ для некоторого $t \geqslant 0$ следует, ито $\xi_{1}=\xi_{2}$.

Действительно, утверждение следствия 3.3 сразу следует из следствия 1.1, примененного к уравнению (3.2).

ТеОРема 3.2. Пусть выполненьи условия предъдущей теоремьь. Тогда разрешающий оператор $S_{t}: V_{0} \rightarrow V_{0}$ дифференцируем по Фреше в пространстве $V_{0}$ при любом фиксированном $t \geqslant 0$ и его производная $w(t)=\mathscr{D}_{u_{0}} S_{t}\left(u_{0}\right) \xi$, $\xi \in V_{0}$, является единственным (согласно теореме 1.1) решением следующей задачи:

$$
\left\{\begin{array}{l}
a\left(\partial_{t}^{2} w+\Delta_{x} w\right)-\gamma \partial_{t} w-f^{\prime}\left(S_{t}\left(u_{0}\right)\right) w=0 \\
\left.w\right|_{t=0}=\xi, \quad\|w\|_{\lambda_{0}}<\infty
\end{array}\right.
$$

Оператор $\Phi: V_{0} \rightarrow V_{0}^{\prime}$ дифференцируем по Фреше, и его производная имеет вид

$$
\mathscr{D}_{u_{0}} \Phi\left(u_{0}\right) \xi=\partial_{t} w(0), \quad w(t)-\text { решение задачи }(3.9) \text { с } w(0)=\xi .
$$

Более того, $S_{t} \in C\left(\mathbb{R}_{+} \times V_{0}, V_{0}\right), \Phi \in C^{1}\left(V_{0}, V_{0}^{\prime}\right)$ и производная Фреше $\mathscr{D}_{u_{0}} S_{t}\left(u_{0}\right)$ равномерно непрерывна по $u_{0} \in B$ для любого ограниченного подмножества $B \subset V_{0}$ и $t \in[T, T+1]$.

ДоказАТЕЛЬСтво. Пусть $u_{01}, u_{02} \in B,\|B\|_{V_{0}} \leqslant M$. Обозначим через $u_{1}(t)$ и $u_{2}(t)$ - решения задач $(0.1)$ с начальньми условиями $u_{01}$ и $u_{02}$ соответственно. Пусть также $v(t)=u_{2}(t)-u_{1}(t)$ и $w(t)$ - решение задачи $(3.9)$ с начальным условием $\xi=u_{02}-u_{01}$. Тогда, как нетрудно проверить, функция $\theta(t)=v(t)-w(t)$ является решением следующего уравнения:

$$
\left\{\begin{array}{l}
a\left(\partial_{t}^{2} \theta+\Delta_{x} \theta\right)-\gamma \partial_{t} \theta-f^{\prime}\left(u_{1}\right) \theta=\left(q_{u_{1}, u_{2}}-f^{\prime}\left(u_{1}\right)\right) v \equiv L(t) v(t), \\
\left.\theta\right|_{t=0}=0, \quad\|\theta\|_{\lambda_{0}}<\infty
\end{array}\right.
$$

Напомним, что функция $q_{u_{1}, u_{2}}$ в правой части уравнения (3.10) определяется формулой (3.3).

Уравнение (3.10) удовлетворяет всем условиям теоремы 1.1, следовательно, согласно оценке (1.8)

$$
\left\|\theta, \Omega_{T}\right\|_{2, p}^{p} \leqslant C e^{p \lambda_{0} T} \int_{\mathbb{R}_{+}} e^{-\alpha|T-s|-p \lambda_{0} s}\|L(s) v(s)\|_{0, p}^{p} d s .
$$

Оценивая $\|v(s)\|_{0, p}$ при помощи неравенства (3.6), получим:

$$
\left\|\theta, \Omega_{T}\right\|_{2, p}^{p} \leqslant C_{1} e^{p \lambda_{0} T}\|v(0)\|_{V_{0}}^{p} \int_{\mathbb{R}_{+}} e^{-\alpha|T-s|-p \alpha s}\|L(s)\|_{0, \infty}^{p} d s .
$$

Таким образом, необходимо доказать, что

$$
Z(\|v(0)\|) \equiv \int_{\mathbb{R}_{+}} e^{-\alpha|T-s|-p \alpha s}\|L(s)\|_{0, \infty}^{p} d s
$$


стремится к нулю при $\|v(0)\|_{V_{0}} \rightarrow 0$.

Так как согласно теореме 2.1 и теореме вложения С. Л. Соболева $\left(W_{2, p} \subset C\right)$ $\left\|u_{i}\right\|_{0, \infty} \leqslant M_{1}=M_{1}\left(\|B\|_{V_{0}}\right)$, то из непрерьвности функции $f^{\prime}$ и определения функции $L$ следует, что $\left\|L, \Omega_{+}\right\|_{0, \infty} \leqslant M_{2}=M_{2}\left(\|B\|_{V_{0}}\right)$.

Фиксируем произвольное $\varepsilon>0$ и выберем $R(\varepsilon)$ так, чтобы

$$
\int_{R(\varepsilon)}^{\infty} e^{-p \alpha s} M_{2}^{p} d s<\frac{\varepsilon}{2}
$$

Тогда из равенства (3.13) после элементарных оценок получим:

$$
Z(\|v(0)\|) \leqslant R(\varepsilon)\left\|L, \Omega_{0, R(\varepsilon)}\right\|_{0, \infty}^{p}+\frac{\varepsilon}{2} .
$$

Так как функция $f^{\prime}$ непрерывна, то существует $\delta_{1}=\delta_{1}(\varepsilon)$ такое, что

$$
\left|f^{\prime}\left(\xi+\xi_{1}\right)-f^{\prime}(\xi)\right|<\left(\frac{\varepsilon}{2 R(\varepsilon)}\right)^{1 / p}
$$

при $|\xi|<2 M_{1}$ и $\left|\xi_{1}\right|<\delta_{1}$. Таким образом, при $\left\|v, \Omega_{0, R(\varepsilon)}\right\|_{0, \infty}<\delta_{1}$

$$
|L(t, x)|^{p} \leqslant \int_{0}^{1}\left|f^{\prime}\left(u_{1}(t, x)+\rho v(t, x)\right)-f^{\prime}\left(u_{1}(t, x)\right)\right|^{p} d \rho \leqslant \frac{\varepsilon}{2 R(\varepsilon)}
$$

при $(t, x) \in \Omega_{0, R(\varepsilon)}$. Значит, $Z\left(\|v(0)\|_{V_{0}}\right)<\varepsilon$ при $\left\|v, \Omega_{0, R(\varepsilon)}\right\|_{0, \infty}<\delta_{1}$. Однако из оценки (3.6) и теоремы вложения следует, что

$$
\left\|v, \Omega_{0, R(\varepsilon)}\right\|_{0, \infty} \leqslant C e^{\lambda_{0} R(\varepsilon)}\|v(0)\|_{V_{0}} .
$$

Поэтому $Z<\varepsilon$ при $\|v(0)\|_{V_{0}}<\delta=\delta_{1} C^{-1} e^{-\lambda_{0} R(\varepsilon)}$. Таким образом, доказана следующая оценка:

$$
\left\|\theta, \Omega_{T}\right\|_{2, p}=e^{\lambda_{0} T} o\left(\|v(0)\|_{V_{0}}\right) \text { при }\|v(0)\|_{V_{0}} \rightarrow 0 .
$$

Дифференцируемость оператора $S_{t}$ в точке $u_{01} \in V_{0}$ следует из неравенства (3.15) и оценки $\|\theta(t)\|_{V_{0}} \leqslant C\left\|\theta, \Omega_{t}\right\|_{2, p}$.

Дифференцируемость оператора $\Phi$ немедленно следует из неравенства (3.15) и оценки $\left\|\partial_{t} \theta(0)\right\|_{V_{0}^{\prime}} \leqslant C\left\|\theta, \Omega_{0}\right\|_{2, p}$. Заметим, что правая часть формулы (3.15) стремится к нулю при $v \rightarrow 0$ равномерно по $u_{01}, u_{02} \in B$ и $t \in[T, T+1]$, следовательно,

$$
\begin{aligned}
& \left\|\left(\mathscr{D}_{u_{0}} S_{t}\left(u_{01}\right)-\mathscr{D}_{u_{0}} S_{t}\left(u_{02}\right)\right) v(0)\right\|_{V_{0}} \\
& \leqslant\left\|u_{2}(t)-u_{1}(t)-\mathscr{D}_{u_{0}} S_{t}\left(u_{02}\right) v(0)\right\|_{V_{0}}+\left\|u_{1}(t)-u_{2}(t)+\mathscr{D}_{u_{0}} S_{t}\left(u_{01}\right) v(0)\right\|_{V_{0}} \\
& \leqslant C\left\|u_{2}-u_{1}-\mathscr{D}_{u_{0}} S_{t}\left(u_{02}\right) v(0), \Omega_{T}\right\|_{2, p}+C\left\|u_{1}-u_{2}+\mathscr{D}_{u_{0}} S_{t}\left(u_{02}\right) v(0), \Omega_{T}\right\|_{2, p} \\
& \leqslant o\left(\|v(0)\|_{V_{0}}\right) .
\end{aligned}
$$

Равномерная непрерьвность $\mathscr{D}_{u_{0}} S_{t}\left(u_{0}\right)$ по $u_{0} \in B$ следует из оценки (3.16). Так как согласно (3.16) семейство операторов $\left\{S_{t}, t \in[T, T+1]\right\}$ равностепенно непрерывно по $u_{0} \in B$, то для доказательства непрерьвности $S_{t}$ по $\left(t, u_{0}\right)$ достаточно проверить непрерывность по $t$ функции $t \rightarrow S_{t}\left(u_{0}\right)$ при фиксированном $u_{0} \in V_{0}$. Но согласно теореме $2.1 u(t)=S_{t}\left(u_{0}\right) \in W_{2, p}\left(\Omega_{T}\right)$ для любого $T \geqslant 0$, а следовательно, по теореме вложения С. Л. Соболева $u \in C\left(\mathbb{R}_{+}, V_{0}\right)$. Теорема 3.2 доказана. 
СлеДСТВИЕ 3.4. Производная Фреше $\mathscr{D}_{u_{0}} S_{t}\left(u_{0}\right)$ удовлетворяет оценке $(0.10)$.

Действительно, применив к уравнению (3.9) теорему 1.1, получим оценку (0.10).

ЗАмЕчАниЕ 3.1. Отметим, что в теореме 3.2 в действительности доказано, что

$$
\|\theta\|_{\lambda_{0}}=o\left(\|v(0)\|_{V_{0}}\right)
$$

То есть отображение $u_{0} \rightarrow S_{t}\left(u_{0}\right)$ является дифференцируемым как отображение из пространства $V_{0}$ в пространство $W_{2, p}^{\lambda_{0}}$ обобшенных функций, имеющих конечную норму (1.4).

Исследуем теперь оператор $\Phi_{\tau}$, определенный в следствии 3.2 . Для этого введем шкалу банаховых пространств $X_{0}=V_{0}^{\prime}, X_{1}=V_{0}, X_{\beta}=\left(-\Delta_{x}\right)^{-\beta / 2} X_{0}$ при $0 \leqslant \beta \leqslant 1$. Можно показать (см., например, [2]), что

$$
X_{\beta}=W_{1-1 / p+\beta, p}(\omega) \cap\left\{\left.u_{0}\right|_{\partial \omega}=0\right\} \text { при } \beta \neq \frac{1}{p} .
$$

ТеОРема 3.3. Пусть выполнены перечисленные выше условия. Тогда нелинейный оператор $\Phi_{\tau}$, определенный в следствии 3.2 , может быть представлен в виде

$$
\Phi_{\tau}(v)=-\left(-\Delta_{x}\right)^{1 / 2} v+F(\tau, v)+G(\tau), \quad v \in V_{0}, \quad \tau \in \mathbb{R}_{+} .
$$

Здесь $G \in C_{b}\left(\mathbb{R}_{+}, X_{0}\right)$, а нелинейный оператор $F(\tau, \cdot) \in C\left(X_{1}, X_{1}\right) \cap C^{1}\left(X_{1}, X_{\beta}\right)$ для некоторого $\beta>0$ при любом фиксированном $\tau \geqslant 0$. Кроме того,

$$
F \in C_{b}\left(\mathbb{R}_{+}, C\left(X_{1}, X_{1}\right)\right) \quad u \quad\left\|F_{v}^{\prime}(\tau, \xi)\right\|_{L\left(X_{1}, X_{\beta}\right)} \leqslant Q\left(\|\xi\|_{X_{1}}\right)
$$

равномерно по $\tau \in \mathbb{R}_{+}$. Здесь $Q: \mathbb{R}_{+} \rightarrow \mathbb{R}_{+}$- некоторая монотонная функиия.

ДокАзАТЕЛЬСтво. Без ограничения общности можно считать, что $f(0)=0$. Представим решение $u(t)$ задачи (0.1) (в которой $g(t)$ заменено на $g(t+\tau)$ ) как сумму двух функций $u(t)=v(t)+\theta(t)$, где функция $v-$ решение задачи

$$
\left\{\begin{array}{l}
a\left(\partial_{t}^{2} v+\Delta_{x} v\right)-\gamma \partial_{t} v=g(t+\tau), \\
\left.v\right|_{t=0}=u_{0}, \quad\|v\|_{b}<\infty
\end{array}\right.
$$

а функция $\theta=u-v$ - решение задачи

$$
\left\{\begin{array}{l}
a\left(\partial_{t}^{2} \theta+\Delta_{x} \theta\right)-\gamma \partial_{t} \theta=f\left(S_{t}\left(u_{0}\right)\right) \\
\left.\theta\right|_{t=0}=0, \quad\|\theta\|_{b}<\infty
\end{array}\right.
$$

Определим операторы $F_{1}\left(\tau, u_{0}\right)$ и $F_{2}\left(\tau, u_{0}\right)$ по формулам

$$
F_{1}\left(\tau, u_{0}\right)=\left.\partial_{t} \theta\right|_{t=0}, \quad F_{2}\left(\tau, u_{0}\right)=\left.\partial_{t} v\right|_{t=0} .
$$


Тогда $\Phi_{\tau}=F_{1}+F_{2}$.

Исследуем сначала оператор $F_{1}$. Так как $f \in C^{1}$ и $f(0)=0$, то согласно оценке $(0.9)\left\|f(u), \Omega_{0,2}\right\|_{1, p} \leqslant Q_{1}\left(\left\|u_{0}\right\|_{V_{0}}\right)$ для некоторой функции $Q_{1}$ и $\left.f(u)\right|_{\partial \omega}=0$. Так как $\theta(0)=0$, то согласно теореме об $\left(W_{3, p}, W_{1, p}^{0}\right)$-регулярности решений уравнения Лапласа аналогично доказательству леммы 1.5 получаем, что $\theta \in W_{3, p}\left(\Omega_{0}\right)$, и справедлива оценка

$$
\left\|\theta, \Omega_{0}\right\|_{3, p} \leqslant C\left\|f(u), \Omega_{0,2}\right\|_{1, p} \leqslant C Q_{1}\left(\left\|u_{0}\right\|_{V_{0}}\right) .
$$

Таким образом, $\partial_{t} \theta \in W_{2, p}\left(\Omega_{0}\right)$, а следовательно, $F_{1}\left(\tau, u_{0}\right)=\partial_{t} \theta(0) \in X_{1}$. Непрерывность оператора $F_{1}$ устанавливается совершенно аналогично доказательству теоремы 3.2. Проверим его $\left(X_{1}, X_{\beta}\right)$-дифференцируемость.

Нетрудно показать, используя результат замечания 3.1 , вложение $W_{2, p}$ в $C$ и дифференцируемость нелинейной функции $f$, что отображение $u_{0} \rightarrow f\left(S_{t}\left(u_{0}\right)\right)$ дифференцируемо как отображение из $X_{1}$ в пространство $L_{q}^{\lambda_{0}}\left(\Omega_{+}\right)$обобщенных функций из $L_{q}^{\mathrm{loc}}\left(\Omega_{+}\right)$, имеющих конечную норму (1.3) (в которой показатель $p$ заменен на $q$ ) для любого $q, 1 \leqslant q \leqslant \infty$. Применив теорему 1.1 к уравнению $(3.22)$, получим, что отображение $\Theta: u_{0} \rightarrow \theta(t)$ дифференцируемо как отображение из $X_{1}$ в $W_{2, q}^{\lambda_{0}}\left(\Omega_{+}\right)$. Фиксируем теперь некоторое $q>p$. Тогда согласно теореме о следах отображение $u_{0} \rightarrow \partial_{t} \theta(0)$ дифференцируемо как отображение из $X_{1}$ в $W_{1-1 / q, q}^{0}(\omega) \subset X_{1 / p-1 / q}$. Итак, оператор $F_{1}(\tau, \cdot)\left(X_{1}, X_{\beta}\right)$-дифференцируем при $\beta=1 / p-1 / q>0$.

Непрерьвность производной $\mathscr{D}_{u} F_{1}$ доказывается так же, как и в теореме 3.2 .

Для доказательства оценки (3.20) рассмотрим уравнение в вариациях, соответствуюшее задаче $(3.22)\left(Q(t)=\mathscr{D}_{u_{0}} F_{1}\left(\tau, u_{0}\right) \xi\right)$ :

$$
\left\{\begin{array}{l}
a\left(\partial_{t}^{2} Q(t)+\Delta_{x} Q(t)\right)-\gamma \partial_{t} Q(t)=f^{\prime}(u(t)) w(t), \\
\left.Q\right|_{t=0}=0, \quad\|Q\|_{\lambda_{0}}<\infty .
\end{array}\right.
$$

Здесь $w(t)$ - решение задачи (3.9), а $u(t)$ - решение уравнения (0.1), в котором $g(t)$ заменено на $g(t+\tau)$. Применив теорему 1.1 к уравнениям $(3.9)$ и $(3.25)$ и использовав оценку (0.9), получим оценку $(3.20)$ для $F_{1}$.

Исследуем теперь оператор $F_{2}\left(\tau, u_{0}\right)$. Согласно теореме 1.1 существует единственное решение $v_{g}$ уравнения $(3.21)$ с нулевыми начальными условиями. Определим функцию $G(\tau)=\partial_{t} v_{g}(0) \in V_{0}^{\prime}$. Тогда, как нетрудно проверить, $G \in C_{b}\left(\mathbb{R}_{+}, V_{0}^{\prime}\right)$. Определим теперь функцию $V_{u_{0}}(t)$ как единственное решение задачи

$$
\left\{\begin{array}{l}
\partial_{t}^{2} V_{u_{0}}+\Delta_{x} V_{u_{0}}=0, \\
\left.V_{u_{0}}\right|_{t=0}=u_{0}, \quad\left\|V_{u_{0}}\right\|_{b}<\infty .
\end{array}\right.
$$

Стандартными методами теории аналитических полугрупп (см. [15]) нетрудно показать, что $V_{u_{0}}(t)=e^{-t\left(-\Delta_{x}\right)^{1 / 2}} u_{0}$. Таким образом, $\partial_{t} V_{u_{0}}(0)=-\left(-\Delta_{x}\right)^{1 / 2} u_{0}$.

И, наконец, рассмотрим функцию $w(t)=v(t)-v_{g}(t)-V_{u_{0}}(t)$, удовлетворяюшую уравнению

$$
\left\{\begin{array}{l}
a\left(\partial_{t}^{2} w+\Delta_{x} w\right)-\gamma \partial_{t} w=\gamma \partial_{t} V_{u_{0}}(t) \\
\left.w\right|_{t=0}=0, \quad\|w\|_{b}<\infty
\end{array}\right.
$$


Так как правая часть $\gamma \partial_{t} V_{u_{0}}(t)$ уравнения (3.27) принадлежит пространству $W_{1, p}^{\text {loc }}\left(\Omega_{+}\right)$, то так же, как и для оператора $F_{1}$, доказывается, что линейный оператор $F_{3}: u_{0} \rightarrow \partial_{t} w(0)$ ограничен как оператор из $X_{1}$ в $X_{1}$. Итак,

$$
F_{2}\left(\tau, u_{0}\right)=-\left(-\Delta_{x}\right)^{1 / 2} u_{0}+F_{3} u_{0}+G(\tau)
$$

Обозначив $F=F_{1}+F_{3}$, получим разложение (3.19). Теорема 3.3 доказана.

Соотношение (3.8) может быть рассмотрено как эволюционное уравнение в пространстве $V_{0}$.

СлЕДСТВИЕ 3.5. Задачи (0.1) и (0.11) әквивалентны.

ДокАЗАТЕЛЬство. Действительно, по построению оператора $\Phi_{\tau}$ всякое решение задачи (0.1) является также и решением задачи (3.8) или, что то же самое, задачи (0.11). Поэтому сушествование решения задачи (0.11) следует из разрешимости задачи (0.1). Таким образом, для доказательства эквивалентности достаточно доказать единственность решения задачи (0.11).

Пусть $u_{1}(t)$ и $u_{2}(t)$ - два решения задачи $(0.11)$ такие, что $u_{1}(0)=u_{2}(0)$. Тогда функция $v(t)=u_{1}(t)-u_{2}(t)$ удовлетворяет следуюшему соотношению (см. [15]):

$$
v(t)=\int_{0}^{t} e^{(s-t)\left(-\Delta_{x}\right)^{1 / 2}}\left(F\left(s, u_{1}(s)\right)-F\left(s, u_{2}(s)\right)\right) d s
$$

Из теоремы 3.3 следует, что $\left\|F\left(s, u_{1}(s)\right)-F\left(s, u_{2}(s)\right)\right\|_{X_{\beta}} \leqslant C\|v(s)\|_{X_{1}}$, а из секториальности оператора $\left(-\Delta_{x}\right)^{1 / 2}$ получаем (см. [16], [17]), что

$$
\left\|e^{(s-t)\left(-\Delta_{x}\right)^{1 / 2}}\right\|_{L\left(X_{\beta}, X_{1}\right)} \leqslant C(t-s)^{\beta-1}
$$

Оценив норму правой части соотношения (3.28) при помощи оценки (3.29), получим:

$$
\|v(t)\|_{X_{1}} \leqslant C \int_{0}^{t}(t-s)^{\beta-1}\|v(s)\|_{X_{1}} d s .
$$

Напомним, что $\beta-1>-1$, поэтому, применив неравенство Гронуолла к оценке (3.30), получим $\|v(t)\|_{X_{1}} \equiv 0$.

СлЕДСТВИЕ 3.6. Задача (3.9) әквивалентна следующей эволюиионной задаче в пространстве $V_{0}$ :

$$
\left\{\begin{array}{l}
\partial_{t} w+\left(-\Delta_{x}\right)^{1 / 2} w=F_{u}^{\prime}(t, u(t)) w \\
\left.w\right|_{t=0}=\xi, \quad w \in C\left(\mathbb{R}_{+}, V_{0}\right) \cap C^{1}\left(\mathbb{R}_{+}, V_{0}^{\prime}\right)
\end{array}\right.
$$

Здесь оператор F определен формулой (3.19), а и(t)-решение задачи (0.1).

Доказательство следствия 3.6 совершенно аналогично доказательству следствия 3.5 . 


\section{§4. Нелинейное эллиптическое уравнение в окрестности гиперболической стационарной точки}

В этом параграфе мы будем изучать автономное уравнение (0.1), т.е. уравнение (0.1) с правой частью, не зависяшей от $t$ :

$$
g(t, x) \equiv g(x) \in L_{p}(\omega) .
$$

В этом случае, как нетрудно проверить, операторы $\left\{S_{t}: V_{0} \rightarrow V_{0}, t \geqslant 0\right\}$ образуют полугруппу в $V_{0}$

$$
S_{t_{1}+t_{2}}=S_{t_{1}} S_{t_{2}}, \quad t_{1}, t_{2} \geqslant 0
$$

Пусть $z_{0}$ - стационарная точка этой полугруппы, т.е. $S_{t} z_{0}=z_{0} \forall t \geqslant 0$ или, что то же самое,

$$
\left\{\begin{array}{l}
a \Delta_{x} z_{0}-f\left(z_{0}\right)=g \\
\left.z_{0}\right|_{\partial \omega}=0
\end{array}\right.
$$

ЗАмечАниЕ 4.1. Аналогично доказательству теорем 2.1 и 2.3, но существенно проше, доказывается, что множество решений системы (4.3) непусто и любое ее решение $z_{0} \in V_{0}$ принадлежит пространству $W_{2, p}(\omega)$.

Согласно теореме 3.2 оператор $S_{t} \in C^{1}\left(V_{0}, V_{0}\right)$ при любом $t \geqslant 0$, следовательно, корректно следующее определение.

ОПрЕДЕЛЕНИЕ 4.1. Стационарная точка $z_{0} \in V_{0}$ полугруппы $S_{t}$ назьвается гиперболической, если спектр производной $\Phi$ реше $\mathscr{D}_{u_{0}} S_{t}\left(z_{0}\right)$ при $t=1$ не пересекается с единичной окружностью

$$
\sigma\left(\mathscr{D}_{u_{0}} S_{1}\left(z_{0}\right)\right) \cap\{\lambda \in \mathbb{C}:|\lambda|=1\}=\varnothing .
$$

Размерность корневого подпространства, соответствуюшего части спектра оператора $\mathscr{D}_{u_{0}} S_{t}\left(z_{0}\right)$, лежащей вне единичной окружности, называется индексом неустойчивости гиперболической точки $z_{0}$

$$
\operatorname{ind}_{z_{0}}=\#\left\{\lambda \in \sigma\left(\mathscr{D}_{u_{0}} S_{1}\left(z_{0}\right)\right),|\lambda|>1\right\} .
$$

ТЕОРема 4.1. Пусть $S_{t}$ - полугруппа, соответствующая автономному уравнению (0.1), $z_{0}$ - ее стачионарная точка. Тогда

1) оператор $\mathscr{D}_{u_{0}} S_{1}\left(z_{0}\right): V_{0} \rightarrow V_{0}$ компактен $и$, следовательно, его спектр дискретен и состоит из нормальных собственных значений конечной кратности;

2) точка $z_{0}$ является гиперболической тогда и только тогда, когда операторный пучок

$L_{z_{0}}(\lambda)=a \lambda^{2}-\gamma \lambda+a \Delta_{x}-f^{\prime}\left(z_{0}\right): W_{2, p}(\omega) \cap W_{1, p}^{0}(\omega) \rightarrow L_{p}(\omega)$

не имеет собственных значений на мнимой оси

$$
\sigma\left(L_{z_{0}}(\cdot)\right) \cap\{\operatorname{Re} \lambda=0\}=\varnothing ;
$$

3) индекс неустойчивости гиперболической точки $z_{0}$ конечен и въчисляется по следующей формуле:

$$
\operatorname{ind}_{z_{0}}=\#\left\{\lambda \in \sigma\left(L_{z_{0}}(\cdot)\right): 0<\operatorname{Re} \lambda<\lambda_{0}\right\}
$$

здесь показатель $\lambda_{0}$ определяется условием (0.8). 
ДокАЗАТЕЛЬСтво. Согласно теореме 3.3 и следствию 3.6 уравнение в вариациях (3.9), определяющее оператор $\mathscr{D}_{u_{0}} S_{1}\left(z_{0}\right)$, может быть записано в виде

$$
\left\{\begin{array}{l}
\partial_{t} w=-\left(-\Delta_{x}\right)^{1 / 2} w+F^{\prime}\left(z_{0}\right) w, \\
\left.w\right|_{t=0}=\xi, w \in C\left([0,1], V_{0}\right) \cap C^{1}\left([0,1], V_{0}^{\prime}\right), \\
\mathscr{D}_{u_{0}} S_{1}\left(z_{0}\right) \xi=w(1) .
\end{array}\right.
$$

Согласно теореме 3.3 оператор $F^{\prime}\left(z_{0}\right)=\mathscr{D}_{u_{0}} F\left(z_{0}\right): X_{1} \rightarrow X_{\beta}$, а следовательно, оператор $F^{\prime}\left(z_{0}\right)$ компактен как оператор из $X_{1}$ в $X_{0}$. Таким образом, согласно теореме о компактных возмушениях секториальных операторов (см. [15]) оператор $A_{z_{0}}=-\left(-\Delta_{x}\right)^{1 / 2}+F^{\prime}\left(z_{0}\right): X_{1} \rightarrow X_{0}$ также является секториальньм и согласно теореме об отображении спектров (см. [18])

$$
\mathscr{D}_{u_{0}} S_{1}\left(z_{0}\right)=e^{A_{z_{0}}} \quad \text { и } \sigma\left(\mathscr{D}_{u_{0}} S_{1}\left(z_{0}\right)\right) \backslash\{0\}=e^{\sigma\left(A_{z_{0}}\right)} .
$$

Так как операторы $\left(-\Delta_{x}\right)^{-1 / 2},\left(-\Delta_{x}\right)^{1 / 2} F^{\prime}\left(z_{0}\right): X_{1} \rightarrow X_{1}$ являются компактными, то, как нетрудно показать (см. [17], [18]), оператор $e^{t A_{z_{0}}}$ компактен при любом $t>0$. Пункт 1) теоремы 4.1 доказан.

Докажем, что справедливо следующее равенство:

$$
\sigma\left(-\left(-\Delta_{x}\right)^{1 / 2}+F^{\prime}\left(z_{0}\right)\right)=\sigma\left(L_{z_{0}}(\cdot)\right) \cap\left\{\operatorname{Re} \lambda<\lambda_{0}\right\}
$$

причем соответствующие собственные векторы и их корневые подпространства также совпадают. Действительно, пусть $\lambda \in \sigma\left(-\left(-\Delta_{x}\right)^{1 / 2}+F^{\prime}\left(z_{0}\right)\right)$ и $z$ - соответствуюший собственный вектор. Тогда функция $w(t)=e^{\lambda t} z$ - решение уравнения (4.9), а значит, согласно следствию 3.6 - и уравнения (3.9), в котором $u_{0}$ заменено на $z_{0}$. Подставив $w(t)$ в уравнение $(3.9)$, получим $e^{\lambda t} L_{z_{0}}(\lambda) z=0$. Таким образом, $z$ - собственный вектор оператора $L_{z_{0}}(\lambda)$. По определению решения системы (3.9) получаем также, что $\operatorname{Re} \lambda<\lambda_{0}$. Обратное включение, а также утверждение о совпадении присоединенных векторов доказывается совершенно аналогично. Итак, формула (4.11) доказана. Пункт 2) теоремы 4.1 немедленно следует из формул (4.10) и (4.11). Докажем третью часть теоремы. Пусть $E_{+}$и $E_{-}-$корневые подпространства оператора $A_{z_{0}}$, соответствуюшие спектральным множествам $\sigma_{+}=\sigma\left(A_{z_{0}}\right) \cap\{\operatorname{Re} \lambda>0\}$ и $\sigma_{-}=\sigma\left(A_{z_{0}}\right) \cap\{\operatorname{Re} \lambda<0\}$. Тогда $V_{0}=E_{+}+E_{-}$и, как нетрудно показать (см. [17]),

$$
\left\|e^{A_{z_{0}} t} z\right\|_{V_{0}} \leqslant C e^{-\varepsilon t}\|z\|_{V_{0}} \forall z \in E_{-} \text {и }\left\|e^{A_{z_{0}} t} z\right\|_{V_{0}} \geqslant C e^{\varepsilon t}\|z\|_{V_{0}} \forall z \in E_{+}
$$

при некотором $\varepsilon>0$. Таким образом, $\operatorname{ind} z_{z_{0}}=\operatorname{dim} E_{+}$. Формула (4.8) сразу следует теперь из равенства (4.11). Теорема 4.1 доказана.

Замечание 4.2. Как известно (см., например, [2]), спектр пучка (4.6) не зависит от $p, 1<p<\infty$, и состоит из нормальных собственных значений конечной кратности. Таким образом, при вычислении индекса неустойчивости ind $z_{0}$ можно считать, что пучок (4.6) действует в гильбертовом пространстве $(p=2)$.

В случае, когда нелинейная функция является потенциальной, можно получить более простую формулу для ind $z_{0}$. 
ТЕОРема 4.2. Пусть нелинейная функиия $f$ удовлетворяет условию (0.13). Тогда индекс неустойчивости стачионарной гиперболической точки $z_{0}$ равен числу положительных собственных значений оператора $а \Delta_{x}-f^{\prime}\left(z_{0}\right)$ (с учетом их кратности):

$$
\operatorname{ind}_{z_{0}}=\#\left\{\lambda \in \sigma\left(a \Delta_{x}-f^{\prime}\left(z_{0}\right)\right): \lambda>0\right\}
$$

Для доказательства этой теоремы нам понадобится следующая лемма.

ЛЕмма 4.1. Пусть выполнены условия предыдущей теоремы и условие (0.13). Тогда спектр операторного пучка (4.6) является вещественным.

ДокАЗАТЕЛЬство. Согласно замечанию 4.2 спектр пучка $L_{z_{0}}$ не зависит от показателя $p, 1<p<\infty$, поэтому достаточно рассмотреть гильбертов случай $p=2$. Пусть $\lambda=\omega+i \alpha \in \sigma\left(L_{z_{0}}\right)$. Тогда, так как спектр $L_{z_{0}}$ является дискретным, то существует $x_{0} \in W_{1,2}^{0}(\omega)$ такой, что

$$
L_{z_{0}}(\lambda) x_{0}=0
$$

Следовательно, согласно условию (0.8) и самосопряженности матрицы $f^{\prime}\left(z_{0}\right)$

$$
\begin{aligned}
0 & <-\left(L_{z_{0}}\left(\lambda_{0}\right) x_{0}, x_{0}\right)=\left(\left[L_{z_{0}}(\lambda)-L_{z_{0}}\left(\lambda_{0}\right)\right] x_{0}, x_{0}\right) \\
& =\left(\lambda^{2}-\lambda_{0}^{2}\right)\left(a x_{0}, x_{0}\right)-\left(\lambda-\lambda_{0}\right)\left(\gamma x_{0}, x_{0}\right) \\
& =\left(\omega^{2}-\alpha^{2}-\lambda_{0}^{2}\right)\left(a x_{0}, x_{0}\right)-\left(\omega-\lambda_{0}\right)\left(\gamma x_{0}, x_{0}\right)+i\left\{2 \alpha \omega\left(a x_{0}, x_{0}\right)-\alpha\left(\gamma x_{0}, x_{0}\right)\right\},
\end{aligned}
$$

или

$$
\begin{gathered}
\alpha\left[\left(\gamma x_{0}, x_{0}\right)-2 \omega\left(a x_{0}, x_{0}\right)\right]=0 \\
\left(\omega^{2}-\alpha^{2}-\lambda_{0}^{2}\right)\left(a x_{0}, x_{0}\right)-\left(\omega-\lambda_{0}\right)\left(\gamma x_{0}, x_{0}\right)>0 .
\end{gathered}
$$

Таким образом, если $\alpha \neq 0$, то $\left(\gamma x_{0}, x_{0}\right)=2 \omega\left(a x_{0}, x_{0}\right)$. Подставляя эту формулу в неравенство (4.15), получим:

$$
\left(\omega^{2}+\alpha^{2}+\lambda_{0}^{2}-2 \omega \lambda_{0}\right)\left(a x_{0}, x_{0}\right)<0
$$

что противоречит положительной определенности оператора $a$. Лемма 4.1 доказана.

Рассмотрим семейство операторных пучков

$$
L^{s}(\lambda)=s L_{z_{0}}(\lambda)+(1-s)\left(\lambda^{2} a_{-} I d-\lambda \gamma_{+} I d+a \Delta_{x}-f^{\prime}\left(z_{0}\right)\right)
$$

где $s \in[0,1]$, а $\gamma_{+}>0$ - достаточно большое положительное число, удовлетворяющее условию $\gamma_{+} \mathrm{Id}-\gamma>0$. 
ОПРЕДЕЛЕНИЕ 4.2. Назовем индексом неустойчивости пучка (4.16) количество его собственных значений пучка (с учетом их кратности), принадлежащих интервалу $\left(0, \lambda_{0}\right)$ :

$$
\text { ind } L^{s}=\#\left\{\sigma\left(L^{s}\right) \cap\left(0, \lambda_{0}\right)\right\} \text {. }
$$

Тогда из условий $(0.8)$ и определения $L^{s}(\lambda)$ следует, что

$$
-\left(L^{s}\left(\lambda_{0}\right) x, x\right)>0 \quad \forall x \in W_{1,2}^{0}(\omega), \quad x \neq 0, \quad s \in[0,1] .
$$

Таким образом, $\lambda_{0} \notin \sigma\left(L^{s}\right)$ при $s \in[0,1]$. Очевидно также, что $0 \notin \sigma\left(L^{s}\right)$ при $s \in[0,1]$, а из леммы 4.1 следует, что спектр пучков $L^{s}$ является вешественным при любом $s \in[0,1]$. Следовательно, согласно теореме об устойчивости корневых кратностей (см. [19]) ind $L^{s}$ не зависит от $s \in[0,1]$. То есть

$$
\operatorname{ind}_{z_{0}}=\operatorname{ind} L_{z_{0}}=\text { ind } L^{0} \text {. }
$$

Таким образом, достаточно проверить утверждение теоремы для пучка

$$
L^{0}(\lambda)=\lambda^{2} a_{-} \operatorname{Id}-\lambda \gamma_{+} \operatorname{Id}+a \Delta_{x}-f^{\prime}\left(z_{0}\right)
$$

ЛЕмма 4.2. Справедливо равенство

$$
\text { ind } L^{0}=\#\left\{\lambda \in \sigma\left(a \Delta_{x}-f^{\prime}\left(z_{0}\right)\right): \lambda>0\right\} \text {. }
$$

ДокАЗАТЕЛЬСТво. Пусть $\left\{e_{i}\right\}_{i=1}^{\infty}-$ полная в $L_{2}$ ортонормированная система собственных векторов самосопряженного оператора $-a \Delta_{x}+f^{\prime}\left(z_{0}\right)$,

$$
\mu_{1} \leqslant \mu_{2} \leqslant \cdots \leqslant \mu_{N}<0<\mu_{N+1} \leqslant \cdots
$$

- соответствующие собственные значения. Тогда, как нетрудно проверить, собственные значения пучка $L^{0}$ вычисляются по следующим формулам:

$$
\lambda_{i}^{+}=\frac{\gamma_{+}+\sqrt{\gamma_{+}^{2}+4 a_{-} \mu_{i}}}{2 a_{-}}, \quad \lambda_{i}^{-}=\frac{\gamma_{+}-\sqrt{\gamma_{+}^{2}+4 a_{-} \mu_{i}}}{2 a_{-}}
$$

а условие (4.17) при $s=0$ эквивалентно следуюшим условиям:

$$
\gamma_{+}^{2}+4 a_{-} \mu_{1}>0, \quad \lambda_{1}^{-}<\lambda_{0}<\lambda_{1}^{+}
$$

Итак, в интервал $\left(0, \lambda_{0}\right)$ попадают собственные значения $\lambda_{i}^{-}$при $i=1, \ldots, N$ и только они. Лемма 4.2 доказана. Теорема 4.2 доказана. 
ОПРЕДЕЛЕНИЕ 4.3. Неустойчивым множеством стационарной точки $z_{0}$ полугрупшы $S_{t}$, определенной в $\S 3$, назьвается следующее множество:

$$
\begin{aligned}
\mathscr{M}^{+}\left(z_{0}\right)=\left\{u_{0} \in V_{0}\right. & : \exists u \in C_{b}\left(\mathbb{R}, V_{0}\right), u(0)=u_{0}, \\
& \left.\left(S_{t} u\right)(s)=u(t+s) \text { такая, что } \lim _{s \rightarrow-\infty} u(s)=z_{0}\right\} .
\end{aligned}
$$

Из определения 4.3 следует, что множество $\mathscr{M}^{+}\left(z_{0}\right)$ является строго инвариантнцм относительно полугруппы $S_{t}$, т.е.

$$
S_{t} \mathscr{M}^{+}\left(z_{0}\right)=\mathscr{M}^{+}\left(z_{0}\right) \text { при } t \geqslant 0 .
$$

ТЕОРема 4.3. Пусть $z_{0}$ - гиперболическая стационарная точка полугрупnы $S_{t}$, и пусть выполнены условия теоремы 4.1. Тогда множсество $\mathscr{M}^{+}\left(z_{0}\right)$ обладает структурой $C^{1}$-многообразия размерности $d=\operatorname{ind}_{z_{0}}$, m.е. существует вложсение $\pi: \mathbb{R}^{d} \rightarrow V_{0}$ класса $C^{1}$ такое, что $\pi\left(\mathbb{R}^{d}\right)=\mathscr{M}^{+}\left(z_{0}\right)$.

Для любого $t \geqslant 0$ сужение оператора $S_{t}$ на многообразие $\mathscr{M}^{+}\left(z_{0}\right)$ является $C^{1}$-диффеоморфизмом.

ДокАЗАТЕЛЬСтво. Для каждого $\delta>0$ аналогично (4.21) определим множество

$$
\begin{array}{r}
\mathscr{M}_{\delta}^{+}\left(z_{0}\right)=\left\{u_{0} \in V_{0}: \exists u \in C\left(\mathbb{R}, V_{0}\right), u(0)=u_{0},\left(S_{t} u\right)(s)=u(t+s)\right. \\
\text { такая, что } \left.\lim _{s \rightarrow-\infty} u(s)=z_{0} \text { и }\|u(s)\|_{V_{0}}<\delta \text { при } s<0\right\} .
\end{array}
$$

Стандартными методами, используя гиперболичность стационарной точки $z_{0}$ и, например, теорему о неявной функции, можно показать, что при достаточно малых $\delta>0$ множество (4.23) является $d=\operatorname{ind}_{z_{0}}$-мерньм $C^{1}$-подмногообразием пространства $V_{0}$, диффеоморфным неустойчивому подпространству $E_{+}$.

Фиксируем $\delta>0$ столь малым, чтобы множество (4.23) являлось $C^{1}$-многообразием, и определим последовательность множеств

$$
\mathbb{M}_{k}^{+}=S_{k}\left(\mathscr{M}_{\delta}^{+}\left(z_{0}\right)\right), \quad k=0,1, \ldots
$$

Тогда согласно определению (4.23)

$$
\mathbb{M}_{k-1}^{+} \subset \mathbb{M}_{k}^{+} \text {и } \mathscr{M}^{+}\left(z_{0}\right)=\bigcup_{k=0}^{\infty} \mathbb{M}_{k}^{+} .
$$

Как показано в [12], из инъективности отображения $S_{k}$ и инъективности его дифференциала следует, что множество $\mathbb{M}_{k}$ диффеоморфно $E_{+}$для любого $k \in \mathbb{N}$. Из представления множества $\mathscr{M}^{+}\left(z_{0}\right)$ в виде (4.25) следует теперь (см. [20]), что множество $\mathscr{M}^{+}\left(z_{0}\right)$ обладает структурой $C^{1}$-многообразия, диффеоморфного $\mathbb{R}^{d}$, $d=\operatorname{ind}_{z_{0}}$. Теорема 4.3 доказана. 
ЗАмЕчАНИЕ 4.3. Отметим, что множество $\mathscr{M}^{+}\left(z_{0}\right)$, вообще говоря, не является $C^{1}$-подмногообразием пространства $V_{0}$, т.е. топологии, задаваемые на множестве $\mathscr{M}^{+}\left(z_{0}\right)$ вложением $\mathscr{M}^{+}\left(z_{0}\right) \subset V_{0}$ и структурой $C^{1}$-многообразия, определяемой представлением $(4.25)$, могут не совпадать $\left(\pi: \mathbb{R}^{d} \rightarrow \mathscr{M}^{+}\left(z_{0}\right)\right.$ не является, вообще говоря, гомеомор физмом). Однако, как показано в [12], в случае, когда полугруппа $S_{t}$ обладает функцией Ляпунова, неустойчивое многообразие $\mathscr{M}^{+}\left(z_{0}\right)$ является $C^{1}$-подмногообразием пространства $V_{0}$. Как будет показано в $\S 5$, полугруппа $S_{t}$ обладает функцией Ляпунова, если выполнено условие (0.13). Таким образом, при вьполнении условия (0.13) неустойчивое множество гиперболической точки $z_{0}-$ $C^{1}$-подмногообразие пространства $V_{0}$ размерности $d=\operatorname{ind}_{z_{0}}$.

\section{§5. Аттрактор нелинейной эллиптической системы в цилиндрической области}

В этом параграфе будет построен аттрактор полугруппы (4.2), соответствующей автономному уравнению (0.1), в пространстве $V_{0}$. Кроме того, в потенциальном случае (в случае выполнения условия (0.13)) доказана регулярность этого аттрактора.

Напомним прежде всего определение аттрактора и достаточные условия его сушествования (подробное изложение см. в [12]).

ОПреДЕЛЕниЕ 5.1. Множество $\mathscr{A}$ назьвается аттрактором полугруппы $S_{t}$, действующей в банаховом пространстве $V_{0}$, если выполнены следующие условия:

(1) $\mathscr{A}$ - компакт в пространстве $V_{0}$;

(2) $\mathscr{A}$ строго инвариантно, т.е. $S_{t} \mathscr{A}=\mathscr{A}$ при $t \geqslant 0$;

(3) $\mathscr{A}$ обладает свойством притяжения ограниченных подмножеств пространства $V_{0}$, т.е. для любого ограниченного $\mathscr{B} \subset V_{0}$

$$
\lim _{t \rightarrow \infty} \operatorname{dist}\left(S_{t} \mathscr{B}, \mathscr{A}\right)=0, \quad \text { где } \operatorname{dist}(X, Y)=\sup _{x \in X} \inf _{y \in Y}\|x-y\|_{V_{0}}
$$

ПРЕДЛОЖЕНИЕ 5.1 (см. [12]). Пусть полугрупnа $S_{t}: V_{0} \rightarrow V_{0}$ непрерьвна при кажсдом фиксированном $t \geqslant 0$, и пусть существует компактное подмножество $K \Subset V_{0}$, обладающее свойством притяэсения ограниченньх подмножеств пространства $V_{0}$. Тогда полугруппа $S_{t}$ обладает аттрактором $\mathscr{A} \subset K$, который может быть представлен в следующем виде:

$$
\begin{aligned}
& \mathscr{A}=\left\{\xi \in V_{0}: \exists u(s), s \in \mathbb{R}, u(0)=\xi\right. \text { такая, ито } \\
& \left.\qquad \sup _{s \in \mathbb{R}}\|u(s)\|_{V_{0}}<\infty u S_{t} u(s)=u(t+s), t \in \mathbb{R}_{+}, s \in \mathbb{R}\right\} .
\end{aligned}
$$

ТеОрема 5.1. Если выполнено условие (4.1), то полугруппа $S_{t}: V_{0} \rightarrow V_{0}$, соответствующая уравнению (0.1), обладает аттрактором $\mathscr{A}$, компактнылм в пространстве $W_{2, p}(\omega)$. 
ДоКАЗАТЕЛЬСТво. Проверим выполнение условий предложения 5.1 для полугруппы $S_{t}$, порождаемой уравнением (0.1). Непрерьвность операторов $S_{t}$ при фиксированных $t \geqslant 0$ доказана в теореме 3.1 . Проверим существование компактного притягивающего множества. Из оценки (0.9) следует, что множество $B=\left\{\zeta \in V_{0}:\|\zeta\|_{V_{0}} \leqslant R\right\}$ является притягивающим множеством полугруппы $S_{t}$ при достаточно большом $R=R\left(\|g\|_{0, p}\right)$, а следовательно, множество $B_{1}=S_{1} B$ также обладает свойством притяжения ограниченных подмножеств пространства $V_{0}$. Докажем, что $B_{1} \subset W_{2, p}(\omega)$. Согласно замечанию 4.1 сушествует стационарная точка $z_{0} \in W_{2, p}(\omega)$ (решение уравнения (4.3)). Умножим уравнение $(0.1)$ на срезаюшую функцию $\varphi(t)$, равную нулю вне отрезка $t \in[0,3]$ и единице на отрезке $t \in[1,2]$, и перепишем его относительно новой неизвестной функции $w=\varphi(t)\left(u-z_{0}\right)$. Получим:

$$
\left\{\begin{array}{l}
\partial_{t}^{2} w+\Delta_{x} w=2 \varphi^{\prime} \partial_{t} u+\varphi^{\prime \prime}\left(u-z_{0}\right)+a^{-1} \varphi\left(f(u)-f\left(z_{0}\right)+\gamma \partial_{t} u\right) \equiv h_{w}(t), \\
\left.w\right|_{\partial \Omega_{0,3}}=0 .
\end{array}\right.
$$

Так как $f \in C^{1}$, то, как нетрудно проверить, $h_{w} \in W_{1, p}^{0}\left(\Omega_{0,3}\right)$ и согласно $(0.9)$ справедлива оценка

$$
\left\|h_{w}, \Omega_{0,3}\right\|_{1, p} \leqslant Q_{1}\left(\left\|u, \Omega_{0,3}\right\|_{2, p}\right) \leqslant Q_{2}\left(\|u(0)\|_{V_{0}}\right) .
$$

Здесь $Q_{1}$ и $Q_{2}$ - некоторые монотонные функции.

Согласно теореме о следах и теореме об $\left(W_{3, p}, W_{1, p}^{0}\right)$-регулярности решений уравнения Лапласа получим:

$$
\left\|u(1)-z_{0}\right\|_{3-1 / p, p} \leqslant C\left\|w, \Omega_{0,3}\right\|_{3, p} \leqslant C_{1}\left\|h_{w}, \Omega_{0,3}\right\|_{1, p} \leqslant C_{1} Q_{2}\left(\left\|u_{0}\right\|_{V_{0}}\right) .
$$

Таким образом, множество $B_{1}-z_{0}$ ограничено в пространстве $W_{3-1 / p, p}(\omega)$, а следовательно, $B_{1} \Subset W_{2, p}(\omega)$. Теорема 5.1 доказана.

СлЕДСТВИЕ 5.1. Пусть $z_{0}$ - гиперболическая стационарная точка полугруппы $S_{t}$. Тогда согласно (5.1)

$$
\mathscr{M}^{+}\left(z_{0}\right) \subset \mathscr{A} .
$$

Таким образом, аттрактор $\mathscr{A}$ содержит инвариантное $C^{1}$-многообразие размерности $d=\operatorname{ind}_{z_{0}}$.

Рассмотрим теперь случай наличия у полугруппы $S_{t}$ функции Ляпунова.

Теорема 5.2. Пусть выполнено условие (0.13). Тогда функиия

$$
-\mathscr{L}(\xi)=\frac{1}{2}(a \Phi(\xi), \Phi(\xi))-\frac{1}{2}(a \nabla \xi, \nabla \xi)-(P(\xi), 1)-(g, \xi): V_{0} \rightarrow R,
$$

где нелинейный оператор $\Phi: V_{0} \rightarrow V_{0}^{\prime}$ определен в следствии 3.2 , а функиия $P$ - в формуле (0.13), удовлетворяет условию

$$
\frac{d}{d t} \mathscr{L}\left(S_{t} \xi\right)=-\left(\gamma \Phi\left(S_{t} \xi\right), \Phi\left(S_{t} \xi\right)\right) \quad \forall \xi \in V_{0}
$$

u, таким образом, является функиией Ляпунова полугруппь $S_{t}$. 
ДокАЗАТЕЛЬСТво. Пусть $u(t)=S_{t} \xi$ - решение задачи (0.1). Тогда согласно определению оператора $\Phi$ имеет место равенство $\Phi(u(t))=\partial_{t} u(t)$, следовательно,

$$
\mathscr{L}(u(t))=\frac{1}{2}(a \nabla u(t), \nabla u(t))-\frac{1}{2}\left(a \partial_{t} u(t), \partial_{t} u(t)\right)+(P(u(t)), 1)+(g, u(t)) .
$$

Продифференцировав функцию (5.5) по $t$ и выразив $\partial_{t}^{2} u(t)$ из уравнения $(0.1)$, получим:

$$
\begin{aligned}
& \frac{d}{d t} \mathscr{L}(u(t))=-\left(\gamma \partial_{t} u(t), \partial_{t} u(t)\right) \\
& \text { или } \\
& \mathscr{L}\left(u\left(t_{2}\right)\right)-\mathscr{L}\left(u\left(t_{1}\right)\right)=-\int_{t_{1}}^{t_{2}}\left(\gamma \partial_{t} u(t), \partial_{t} u(t)\right) d t .
\end{aligned}
$$

Формула (5.4) доказана.

Так как $\gamma>0$, то из (5.6) следует, что $\mathscr{L}\left(S_{t} \xi\right) \leqslant \mathscr{L}(\xi)$, причем знак равенства возможен лишш в случае $\partial_{t} u(s) \equiv 0 \forall s \in[0, t]$, т.е. $\xi$ - стационарная точка полугрупшы $S_{t}$. Таким образом, $\mathscr{L}$ - функция Ляпунова полугруппы $S_{t}$. Теорема 5.2 доказана.

Предположим теперь, что множество стационарных точек полугрупшы $S_{t}$

$$
\mathscr{R}=\left\{z_{i} \in V_{0}: i=1, \ldots, N ; z_{i} \text { - решение задачи (4.3) }\right\}
$$

конечно. Тогда справедлива следуюшая теорема.

ТЕОРЕМА 5.3. Пусть выполненъ условия предыдущей теоремы, и пусть множество (5.7) конечно. Тогда для любого решения $u(t)$ уравнения $(0.1)$ $\left(t \in \mathbb{R}, u \in C_{b}\left(\mathbb{R}, V_{0}\right)\right)$ существуют $z_{+} \in \mathscr{R}$ u $z_{-} \in \mathscr{R}$ такие, ито $z_{-} \neq z_{+} u$

$$
\lim _{t \rightarrow+\infty}\left\|u(t)-z_{+}\right\|_{V_{0}}=0, \quad \lim _{t \rightarrow-\infty}\left\|u(t)-z_{-}\right\|_{V_{0}}=0 .
$$

Доказательство теоремы 5.3 приведено, например, в [4].

СЛЕДСТВИЕ 5.2. Пусть $\mathscr{M}\left(z_{i}\right)$ - неустойчивое множество стационарной точки $z_{i} \in \mathscr{R}$, определенное формулой (4.21). Тогда из равенства (5.8) и представления аттрактора $\mathscr{A}$ в виде (5.1) следует, ито

$$
\mathscr{A}=\bigcup_{i=1}^{N} \mathscr{M}^{+}\left(z_{i}\right) .
$$

TЕОРема 5.4 (регулярность эллиптического аттрактора). Пусть полугруппа $S_{t}: V_{0} \rightarrow V_{0}$, соответствующая уравнению (0.1), обладает непрерывной функиией Ляпунова $\mathscr{L}$, и пусть все ее стационарнье точки (5.7) являются гиперболическими. Тогда аттрактор $\mathscr{A}$ системы (0.1) представим в виде (5.9), причем неустойчивые множества $\mathscr{M}^{+}\left(z_{i}\right)$ являются $C^{1}$-подмногообразиями пространства $V_{0}$ размерности $d_{i}=\operatorname{ind}_{z_{i}}$, диффеоморфныцми $\mathbb{R}^{d_{i}}$.

Доказательство теоремы 5.4 вытекает из формулы (5.9) и теоремы 4.3. 
СлЕДСТВИЕ 5.3. Предположим, что выполнены условия теоремы 5.4. Пусть стачионарнье точки (5.7) занумеровань в порядке возрастания функции Ляпунова, причем для простоть будем считать, что

$$
\mathscr{L}\left(z_{1}\right)<\mathscr{L}\left(z_{2}\right)<\cdots<\mathscr{L}\left(z_{N}\right)
$$

u nycmb

$$
\mathscr{A}_{k}=\bigcup_{i=1}^{k} \mathscr{M}^{+}\left(z_{i}\right) .
$$

Тогда, рассуждая аналогично [12], можно показать, что справедливы следующие утверждения:

1) $\mathscr{A}_{k}$ устойчиво относительно $S_{t}$, т.е. для любой окрестности $\mathscr{O}_{1}\left(\mathscr{A}_{k}\right)$ множества $\mathscr{A}_{k}$ в пространстве $V_{0}$ найдется окрестность $\mathscr{O}_{2}\left(\mathscr{A}_{k}\right)$ такая, что

$$
S_{t} \mathscr{O}_{2}\left(\mathscr{A}_{k}\right) \subset \mathscr{O}_{1}\left(\mathscr{A}_{k}\right) \quad \forall t \geqslant 0
$$

2) граница множества $\mathscr{M}^{+}\left(z_{i}\right)$ строго инвариантна относительно действия полугруппь $S_{t}$, m.e. $S_{t} \partial \mathscr{M}^{+}\left(z_{i}\right)=\partial \mathscr{M}^{+}\left(z_{i}\right)$; более того, $\partial \mathscr{M}^{+}\left(z_{i}\right) \subset \mathscr{A}_{i-1}$

3) $\lim _{t \rightarrow \infty} \operatorname{dist}\left(S_{t} K, \mathscr{A}_{i-1}\right)=0$ для любого компакта $K \subset \mathscr{A}_{i} \backslash z_{i}$.

ЗАмечание 5.1. Как показано, например, в [12], условие гиперболичности всех стационарных точек является условием общего положения, т.е. множество правых частей $g$ уравнения (0.1), удовлетворяющих этому условию, является открытым, всюду плотным подмножеством пространства $L_{p}(\omega)$. Кроме того, в этом случае из теории степени Лере-Шаудера следует, что количество стационарных точек нечетно: $N=2 m+1$.

Исследуем теперь характер притяжения к аттрактору $\mathscr{A}$. Для этого нам понадобится следующее определение

ОПРЕДЕЛЕНИЕ 5.2. Пусть вьполнены условия следствия 5.3. Конечномерной составной траекторией (к.с.т.) полугруппы $S_{t}$ в пространстве $V_{0}$ называется кусочно непрерывная функция $\hat{u}(t), t \in[0, \infty]$, обладаюшая следуюшими свойствами:

1) существуют $0=t_{0}<t_{1}<\cdots<t_{m}<t_{m+1}=+\infty$ такие, что

$$
\hat{u} \in C\left(\left[t_{i}, t_{i}+1\right), V_{0}\right), \quad i=0, \ldots, m ;
$$

2) $\hat{u}(t)=S_{t-t_{i}} u\left(t_{i}\right)$ при $t \in\left[t_{i}, t_{i+1}\right)$;

3) $\hat{u}\left(t_{i}\right) \in \mathscr{M}^{+}\left(z_{\pi(i)}\right)$, где $\pi:\{1, \ldots, m+1\} \rightarrow\{1, \ldots, N\}$.

Таким образом, к.с.т. $\hat{u}(t)$ является кусочно непрерьвной кривой, составленной из конечного числа кусков непрерывных траекторий полугруппы $S_{t}$, лежащих на неустойчивых множествах $\mathscr{M}^{+}\left(z_{i}\right)$. 
ТЕОРема 5.5. Пусть выполнены условия следствия 5.3. Тогда для любого $u_{0} \in V_{0}$ существует к.с.т. $\hat{u}(t)$ такая, что

$$
\left\|S_{t} u_{0}-\hat{u}(t)\right\|_{V_{0}} \leqslant C e^{-\nu t}, \quad t \geqslant 0
$$

Более того, константа $C$ мохет быть въцрана равномерно по $u_{0} \in K$, әде $K$ - произвольный компакт в $V_{0}$, а константа $\nu>0$ зависит лишь от nолугруппьь $S_{t}$.

Доказательство этой теоремы заключается в проверке условий следующей абстрактной теоремы о равномерной спектральной асимптотике (см. [12]).

Теорема 5.6 [12]. Предположим, что все стационарные точки полугрупnвь $S_{t} \in C\left(\mathbb{R}_{+} \times V_{0}, V_{0}\right)$, действующей в некотором банаховом пространстве $V_{0}$, являются гиперболическими. Пусть такжсе полугруппа $S_{t}$ обладает аттрактором $\mathscr{A}$ в $V_{0}$, функиией Ляпунова $\mathscr{L} \in C\left(V_{0}, R\right)$, и пусть для любого ограниченного множества $B \subset V_{0}$ выполнено условие

$$
\left\|\mathscr{D}_{\xi} S_{t}(\xi)\right\|_{L\left(V_{0}, V_{0}\right)} \leqslant C e^{\alpha t} \quad \forall \xi \in B, \quad C=C(B), \quad \alpha=\alpha(B)
$$

Кроме того, пусть при любом фиксированном $t$ производная $\mathscr{D}_{\xi} S_{t}(\xi)$ равномерно непрерывна на любом ограниченном множестве $\xi \in B \subset V_{0}$. Тогда для полугруппь $S_{t}$ справедливо утвержсдение теореми 5.5 .

Все условия теоремы 5.6 в случае полугрупшы $S_{t}$, соответствующей уравнению (0.1), проверены в теоремах $3.3,4.1,5.1,5.2$.

ЗАмЕчАнИЕ 5.2. В формулировке теоремы 5.6 в работе [12] дополнительно требуется, чтобы $S_{t} \in C^{1+\alpha}\left(V_{0}, V_{0}\right)$. Это условие используется лишь для построения неустойчивых многообразий $\mathscr{M}^{+}\left(z_{i}\right)$. Однако для того чтобы $\mathscr{M}^{+}\left(z_{i}\right)$ было $C^{1}$-многообразием, достаточно выполнения условий теоремы 5.6. Доказательство этого факта проводится аналогично доказательству теоремы 4.3.

СлЕДСТВИЕ 5.4. Пусть выполнены условия теоремы 5.5. Тогда для любого ограниченного множества $B \subset V_{0}$ справедлива оценка

$$
\operatorname{dist}\left(S_{t} B, \mathscr{A}\right) \leqslant C(B) e^{-\nu t}
$$

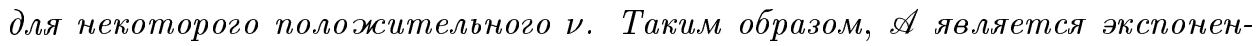
ииальным аттрактором полугруппь $S_{t}$.

Действительно, в случае компактного $B$ формула (5.12) немедленно следует из оценки (5.11). Однако, как показано в доказательстве теоремы 5.1, множество $S_{1} B$ предкомпактно для любого ограниченного $B \subset V_{0}$. Таким образом, оценка (5.12) справедлива и для любого ограниченного $B \subset V_{0}$. 
ЗАмЕЧАНИЕ 5.3. Системы вида (0.1) возникают в приложениях, например, при исследовании решений вида бегушей волны для соответствующих эволюционных уравнений. Так, задача нахождения решений вида $A(t, x)=A\left(x_{1}-\gamma t, x^{\prime}\right)$, $x=\left(x_{1}, x^{\prime}\right) \in \Omega_{+}, t \in \mathbb{R}_{+}, \gamma \in \mathbb{R}$, для обобщенного уравнения Гинзбурга-Ландау

$$
\partial_{t} A=\mu A-(\lambda-i \beta) A|A|^{2}+\Delta_{x} A
$$

в котором $A=A_{1}+i A_{2}, \lambda, \beta, \mu \in \mathbb{R}, \lambda>0$, сводится, как нетрудно проверить, к нахождению всех ограниченных при $t \in \mathbb{R}$ решений уравнения вида $(0.1)$ в полном цилиндре $\Omega=\mathbb{R} \times \omega$, т.е. к нахождению аттрактора уравнения вида $(0.1)$ в полуцилиндре $\Omega_{+}$(см. формулу (5.1)). Отметим также, что при $\beta=0$ это уравнение является потенциальньм.

\section{Список литературы}

1. Соболев С. Л. Некоторые приложения функционального анализа в математической физике. М.: Наука, 1988.

2. Трибель $X$. Теория интерполяции, функциональные пространства, диффференциалњные операторы. М.: Мир, 1980.

3. Бабин A. B. Аттрактор обобщенной полугруппы, порожденной эллиптическим уравнением в цилиндрической области // Изв. РАН. Сер. матем. 1994. Т. 58. № 2. С. 3-18.

4. Вишик М.И., Зелик C. В. Траекторный аттрактор нелинейной эллиптической системы в цилиндрической области // Матем. сб. 1996. Т. 187. № 12. С. 21-56.

5. Зелик C. В. Ограниченность решений нелинейной эллиптической системы в цилиндрической области // Матем. заметки. 1997. Т. 61. №3. С. 447-450.

6. Babin A. V. Inertial manifolds for travelling-wave solutions of reaction-diffusion systems // Comm. Pure Appl. Math. 1995. V. 48. № 2. P. 167-198.

7. Brunovsky P., Mora X., Polacik P., Sola-Morales J. Asymptotic behavior of solutions of semilinear elliptic equations on an unbounded strip // Acta Math. Univ. Comenian. 1991. V. 60. № 2. P. 163-183.

8. Calsina A., Mora X., Sola-Morales J. The dynamical approach to elliptic problems in cylindrical domains, and a study of their parabolic singular limit // J. Differential Equations. 1993. V. 102. № 2. P. 244-304.

9. Kirchgässner K. Wave-solutions of reversible systems and applications // J. Differential Equations. 1982. V. 45. P. 113-127.

10. Mielke A. Essential manifolds for an elliptic problem in an infinite strip // J. Differential Equations. 1994. V. 110. № 2. P. 322-355.

11. Scheel A. Existence of fast travelling waves for some parabolic equations: A dynamical systems approach // J. Dynam. Differential Equations. 1996. V. 8. № 4. P. 469-548.

12. Бабин А. В., Вишик М.И. Аттракторы эволюционных уравнений. М.: Наука, 1989.

13. Hale J. K. Asymptotic behaviour of dissipative systems // Math. Surveys Monographs. 1988. V. 25.

14. Agmon S., Nirenberg L. Lower bounds and uniqueness theorems for solutions of differential equations in a Hilbert space // Comm. Pure Appl. Math. 1967. V. 20. P. 207-229.

15. Клемент $\Phi .$, Хейманс X., Ангенент $A$. и др. Однопараметрические полугруппы. М.: Мир, 1992.

16. Иосида К. Функциональный анализ. М.: Мир, 1967.

17. Хенри Д. Геометрическая теория полулинейных параболических уравнений. М.: Мир, 1985.

18. Davies E. One-parameter semigroups. London: Academic Press, 1980. 
19. Гохберг И., Крейн $М$. Введение в теорию линейных несамосопряженных операторов. М.: Наука, 1965.

20. Хири М. Дифференциальная топология. М.: Мир, 1979.

Институт проблем передачи информации РАН;

Московский государственнњй университет им. М. В. Ломоносова
Поступила в редакцию 20.11 .1998 